\title{
A Dynamic Factor Approach to Nonlinear Stability Analysis
}

by

Mototsugu Shintani

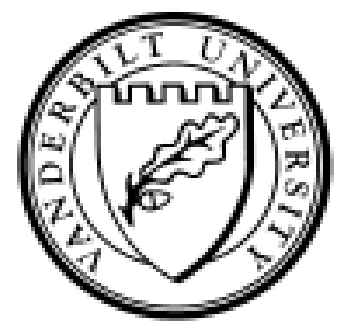

Working Paper No. 04-W18

August 2004

\section{DEPARTMENT OF ECONOMICS \\ VANDERBILT UNIVERSITY \\ NASHVILLE, TN 37235}

www.vanderbilt.edu/econ 


\title{
A Dynamic Factor Approach to Nonlinear Stability Analysis*
}

\author{
Mototsugu Shintani ${ }^{\dagger}$
}

This version: August 2004

\begin{abstract}
A method of principal components is employed to investigate nonlinear dynamic factor structure using a large panel data. The evidence suggests the possibility of nonlinearity in the U.S. while it excludes the class of nonlinearity that can generate endogenous fluctuation or chaos.

Keywords: Chaos; Dynamic Factor Model; Lyapunov Exponents; Nonparametric Regression; Principal Components.

JEL classification: C14; C33
\end{abstract}

\footnotetext{
*An earlier version of this paper was entitled "A Nonlinear Factor Analysis of Business Cycles with a Large Data Set." The author would like to thank Yanqin Fan, Mark Watson and seminar participants at the 2002 NBER Summer Institute, the Common Features in Rio conference, the twelfth annual symposium of the Society for Nonlinear Dynamics and Econometrics, the Duke/NCSU/UNC Triangle Econometrics Workshop, the 2004 Far Eastern Meeting of the Econometric Society, and Vanderbilt University for their helpful comments and discussions.

${ }^{\dagger}$ Department of Economics, Vanderbilt University, Nashville, TN 37235, USA. e-mail: mototsugu.shintani@vanderbilt.edu
} 


\section{Introduction}

Traditionally, we treat expansions and contractions of the economy as a result of exogenous random shocks explained by a change in policy, a change in demand, technological change, and other supply shocks. An alternative view is to consider the endogenous aggregate fluctuation via a chaotic system, or a simple nonlinear deterministic system that can have stochastic-like unpredictable behavior. One of the most convenient empirical methods for considering two competing views of the business cycle is to compute a stability measure called the largest Lyapunov exponent. It measures the sensitive dependence on initial conditions and is often employed to define a chaotic behavior in either a deterministic or stochastic nonlinear system (Eckmann and Ruelle, 1985, and Nychka, Ellner, Gallant, and McCaffrey, 1992). A chaotic system has a positive Lyapunov exponent while an exogenous system with a unique and globally stable steady state has a negative Lyapunov exponent.

In practice, the nonparametric regression method is often employed to compute Lyapunov exponents of an unknown nonlinear autoregressive (AR) model. Such a nonparametric approach was first considered by Eckmann and Ruelle (1985) and Eckmann, Kamphorst, Ruelle, and Ciliberto (1986) and its statistical properties are later considered by McCaffrey, Ellner, Gallant, and Nychka (1992), Whang and Linton (1999), and Shintani and Linton (2004). By applying this nonparametric method to the GDP series from OECD countries, Shintani and Linton (2003) found that the Lyapunov exponents were significantly negative for most cases, which supported the exogenous view of a business cycle as opposed to the chaotic view. This approach is also applied to foreign exchange rates by Dechert and Gençay (1992), monetary 
aggregates by Serletis (1995) and Barnett, Gallant, Hinich, Jungeilges, Kaplan, and Jensen (1995), and the stock return series by Abhyankar, Copeland, and Wong (1997) and Shintani and Linton (2004). However, one potential drawback of the approach employed in these studies is that only a univariate time series could be considered in each estimation. If the true system consists of $N$ equations, the theoretical result known as Takens embedding implies that $2 N+1$ lags are required in the nonlinear AR model for this univariate method to be valid. Therefore, when $N$ is large, the nonparametric estimation method is known to be subject to computational difficulties (the curse of dimensionality).

The main objective of this paper is to consider an alternative approach to conducting a test regarding the stability of the multivariate nonlinear system with a large $N$. Instead of running a nonparametric regression using each individual economic time series, we consider a multiple time series generated from a dynamic factor model with a common factor following a nonlinear process. Such a common factor approach achieves dimensional reduction by construction and thus is less subject to a high dimensionality problem, unlike the nonparametric regression applied to a single series. For this reason, introducing an assumption of the common factor structure in a system of equations seems to be advantageous in a stability analysis of business cycles.

The dynamic factor model, originally considered by Sargent and Sims (1977) and Geweke (1977), has long been employed in macroeconomic analysis, including construction of business cycle indexes, analysis of stock price dynamics and international business cycle analysis. The dynamic factor model also fits well within the framework of the dynamic stochastic general equilibrium (DSGE) models, the workhorse in 
the modern macroeconomics literature, since they predict that a small set of driving forces is responsible for covariation in macroeconomic variables. Furthermore, recent theoretical studies revealed that chaotic endogenous fluctuation can be derived under a DSGE-type framework (e.g., Brock and Hommes, 1998). In this paper, we consider the possibility of applying the Lyapunov exponent-based test to the estimated common factors for the purpose of determining whether these sources of business cycles are better explained using endogenous or exogenous models.

Our testing procedure consists of two steps. We first estimate the common factors in the dynamic factor model by employing the method of principal components and then test the nonlinear factor structure and the chaotic behavior using the estimated factors. The estimator based on principal components has become increasingly popular in the recent literature of dynamic factor models (e.g., Stock and Watson, 2002a,b, Bai, 2003, Diebold, 2003, and Shintani, 2004). We emphasize that the principal component approach is indeed useful in a high dimensional nonlinear framework because it remains theoretically valid under a very flexible nonlinear dynamic factor structure when both the number of the series $(N)$ and the time series observations $(T)$ are large. To investigate this theoretical prediction, we conduct a Monte Carlo simulation using the data generated from both chaotic and stable nonlinear models. The results of the simulation suggest that the method works well for many combinations of $N$ and $T$ typically available in practice. To provide empirical evidence regarding the nonlinear dynamics and the stability, we utilize the full balanced panel of 159 monthly U.S. macroeconomic time series $(N=159)$ with the sample period from 1959:3 to 1998:12 $(T=478)$, and estimate a large dimensional dynamic factor model. The evidence suggests the possibility of nonlinearity in the U.S. while it excludes the 
class of nonlinearity that can generate endogenous fluctuation or chaos. This evidence suggests the justification of using the nonlinear impulse response analysis proposed by Gallant, Rossi, and Tauchen (1993), and Potter (2000); thus, we also report the nonlinear impulse response functions of the common factors.

The remainder of the paper is organized as follows: Section 2 introduces a dynamic factor model and the Lyapunov exponent of a common factor. Simulation results to evaluate the finite sample performance of the method employed in this paper are provided in Section 3. Section 4 presents the empirical results of a nonlinear stability analysis using the large U.S. data. Some concluding remarks are made in Section 5.

\section{Model}

Let $x_{i t}$ be an $i$-th component of $N$-dimensional multiple time series $X_{t}=\left(x_{1 t}, \ldots, x_{N t}\right)^{\prime}$ and $t=1, \ldots, T$. A simple dynamic factor model associates each $x_{i t}$ with a scalar common factor $f_{t}$ in equations

$$
x_{i t}=b_{i} f_{t}+e_{i t}, \quad i=1, \ldots, N \text {, }
$$

where $b_{i}$ 's are factor loadings with respect to $i$-th series, $e_{i t}$ 's are idiosyncratic shocks. A conditional mean function of the common factor $f_{t}$ is assumed to have a $p$-th order nonlinear AR form,

$$
f_{t}=m\left(f_{t-1}, \ldots, f_{t-p}\right)+u_{t}
$$

where $u_{t}$ is a martingale difference sequence with $E\left(u_{t} \mid \mathcal{F}_{t-1}\right)=0$ and $E\left(u_{t}^{2} \mid \mathcal{F}_{t-1}\right)=\sigma_{t}^{2}$ where $\mathcal{F}_{t-1}=\left\{f_{t-1}, f_{t-2}, \ldots\right\}$. Depending on the shape of the nonlinear function $m\left(f_{t-1}, \ldots, f_{t-p}\right)$, the dynamics of $f_{t}$ can be either chaos or stable. The largest Lya- 
punov exponent of the common factor $f_{t}$ is defined as

$$
\lambda \equiv \lim _{M \rightarrow \infty} \frac{1}{2 M} \ln \left|\nu_{1}(M)\right|
$$

where $\nu_{1}(M)$ is the largest eigenvalue of $\mathbf{T}_{M}^{\prime} \mathbf{T}_{M}$, and $\mathbf{T}_{M}=J_{M-1} \cdot J_{M-2} \cdots J_{0}$ where

$$
J_{t}=\left[\begin{array}{ccccc}
\Delta m_{1 t} & \Delta m_{2 t} & \cdots & \Delta m_{p-1, t} & \Delta m_{p t} \\
1 & 0 & \cdots & 0 & 0 \\
0 & 1 & \cdots & 0 & 0 \\
\vdots & \vdots & \ddots & \vdots & \vdots \\
0 & 0 & \cdots & 1 & 0
\end{array}\right]
$$

for $t=0,1, \ldots, M-1$, where $\Delta m_{j t}=\partial m\left(\mathbf{f}_{t}\right) / \partial f_{t-j}$, for $j=1, \ldots, p$, are partial derivatives of conditional mean function evaluated at $\mathbf{f}_{t}=\left(f_{t-1}, \ldots, f_{t-p}\right)$. A chaotic system has a positive Lyapunov exponent while a exogenous system with a unique and globally stable steady state has a negative Lyapunov exponent.

When factor $f_{t}$ is observable, the conditional mean function in equation (2) can be directly estimated using a nonparametric regression method applied to $f_{t}$. The result can then be used to construct a consistent estimator of $\lambda$ following the approach used by Nychka, Ellner, Gallant, and McCaffrey (1992) and others. However, since $f_{t}$ is not observable in the dynamic factor model, the direct estimation is not feasible.

One may want to employ a maximum likelihood estimator (MLE) that incorporates latent variables under some distributional assumptions on $e_{i t}$ 's and $u_{t}$, the approach used by Stock and Watson (1989), Chauvet (1998), and Kim and Nelson (1998) in their analysis of the business cycle index. However, the MLE is not suitable in the current context because of the following two reasons. First, the MLE approach allows nonlinearity but the functional form in equation (2) needs to be specified. The parametric approach of Lyapunov exponent estimation has been considered in some cases (e.g., Bask and de Luna, 2002). However, in practice, information on the 
functional form is usually not available, and therefore the nonparametric approach is generally preferable in the estimation of Lyapunov exponent. Second, the MLE approach becomes computationally difficult when the number of the series $(N)$; thus, the number of parameters becomes large.

In this paper, we first estimate the unobservable common factor $f_{t}$ nonparametrically based on the principal components of $N$ variables instead of relying on the MLE. We then run a nonparametric regression of equation (2) using the estimated factor and compute $\lambda$ just as in the case when $f_{t}$ is observable. Estimation of the latent factor based on principal components has become increasingly popular in the recent literature of dynamic factor models (e.g., Stock and Watson, 2002a,b, Bai, 2003, Diebold, 2003, and Shintani, 2004). We emphasize that this approach is advantageous for the nonlinear stability analysis in a dynamic factor framework since the estimator of the factor remains consistent under a very flexible nonlinear dynamic factor structure when the number of the series $(N)$ and the time series observations (T) are large.

Our approach contrasts with the case of $N$ dimensional system without assuming a common factor structure. In such a case, embedding is required to estimate the Lyapunov exponents (see Gençay and Dechert, 1992, for example). Suppose the dynamics of $X_{t}=\left(x_{1 t}, \ldots, x_{N t}\right)^{\prime}$ are given by $X_{t}=G\left(X_{t-1}\right)$ where $G: R^{N} \rightarrow R^{N}$. In addition, suppose a single time series $y_{t}=w\left(X_{t}\right)$ where $w: R^{N} \rightarrow R$ will be used for the nonlinear stability analysis. The Lyapunov exponent of $G$ is known to be preserved in the embedded dynamics of $y_{t}$ given by $y_{t}=m\left(y_{t-1}, \ldots, y_{t-p^{*}}\right)$ where $p^{*}$ is an embedding dimension that satisfies $p^{*} \geq 2 N+1$. Therefore, computing $\lambda$ of $N$ dimensional system based on nonlinear AR model of a single series $y_{t}$ is justifiable as 
long as $p^{*}$ lags are included. However, one limitation is that $N$ needs to be small to employ a nonparametric method. As $N$ becomes large, the nonparametric regression quickly becomes difficult since $p^{*}$ depends on $N$ (the curse of dimensionality). Our dynamic factor approach, in contrast, is not subject to this problem since the lag length $p$ in equation (2) is assumed to be fixed regardless of the size of $N$.

The remaining issue is to see whether we can justify replacing the true factor with the estimated factor in the nonparametric regression. A related issue in the nonparametric kernel estimator for time series was also considered by Andrews (1995). He derived the conditions required for the generated regressor that depends on the common $\sqrt{T}$-consistent estimator to become negligible in the limiting distribution of nonparametric regression. In the rest of this section, we discuss that a similar argument can be also applied to the nonparametric estimation of factor dynamics.

Our estimator of a common factor, $\widetilde{f}_{t}$, is the first principal component of $N$ series which corresponds to the first eigenvector of the $T \times T$ matrix $X X^{\prime}$ with normalization $T^{-1} \sum_{t=1}^{T} \widetilde{f}_{t}^{2}=1$, where $X$ is the $T \times N$ data matrix with $t$-th row given by $X_{t}^{\prime}=\left(x_{1 t}, \cdots, x_{N t}\right)$. The asymptotic theory of $\tilde{f}_{t}$ under $N, T \rightarrow \infty$ is developed by Stock and Watson (2002b) and Bai (2003) and others. Under mild conditions on moments and memory, the estimator $\tilde{f}_{t}$ has been shown to be a $\sqrt{N}$ consistent estimator of $f_{t}$ (up to a scaling constant). Below is a set of assumptions typically employed in these theoretical studies. Note that const. implies 'for some finite positive constant.'

Assumption F: (i) $E\left(f_{t}\right)=\mu_{f}=0, E\left(\left(f_{t}\right)^{2}\right)=\Sigma_{f}=1, E\left(\left(f_{t}\right)^{4}\right) \leq$ const. and $\sqrt{T}\left(F^{\prime} F / T-\Sigma_{f}\right)=O_{p}(1)$ where $F=\left[f_{1}, \cdots, f_{T}\right]^{\prime}$. 
(ii) $\left|b_{i}\right| \leq$ const. and $\sqrt{N}\left(B^{\prime} B / N-\Sigma_{b}\right)=O_{p}(1)$ where $B=\left[b_{1}, \cdots, b_{N}\right]^{\prime}$.

(iii) $E\left(e_{i t}\right)=0, E\left|e_{i t}{ }^{8}\right| \leq$ const., $E\left(e_{i s} e_{i t}\right)=0$ for all $t \neq s, \bar{\sigma}_{e}^{2}=N^{-1} \sum_{i=1}^{N} \sigma_{e i}^{2} \leq$ const., and $E\left(e_{i t} e_{j t}\right)=\tau_{i j} \leq$ const. for all $t, i$ and $j$.

(iv) $\left\{f_{t}\right\},\left\{b_{i}\right\}$ and $\left\{e_{i t}\right\}$ are mutually independent.

(v) $N / T^{2} \rightarrow \infty$ as $T \rightarrow \infty$.

(i) and (ii) are the moment conditions for the factor $f_{t}$ and factor loading $b_{i}$, respectively. (iii) is a condition on the cross-sectional correlation of the idiosyncratic error $e_{i t}$. This allows the dynamic factor model to have an approximate factor structure instead of an exact factor structure. Serial correlation of $e_{i t}$ can be also incorporated but is not allowed here. (iv) and (v) are stronger than necessary but are introduced here to simplify the derivation of the theoretical result to be followed.

In principle, any nonparametric estimator can be used to estimate equation (2). Here, we follow Andrews (1995) and restrict our attention to Nadaraya-Watson type kernel regression estimator with $p=1$ given by

$$
\widehat{m}(f)=\sum_{t=1}^{T} f_{t} K\left(\frac{f_{t-1}-f}{h}\right) / \sum_{t=1}^{T} K\left(\frac{f_{t-1}-f}{h}\right)
$$

where $K$ is a kernel function and $h$ is a bandwidth such that $h \rightarrow 0$ as $T \rightarrow \infty$. This is an infeasible estimator since $f_{t}$ is not observable. The feasible estimator that replaces $f_{t}$ with $\widetilde{f}_{t}$ will be denoted by $\widetilde{m}(f)$. To derive the asymptotic properties of the nonparametric estimator, we introduce the following assumptions.

Assumption K: (i) $m(f)$ is twice continuously differentiable for all $f$.

(ii) The kernel function $K$ is symmetric around zero, $\int K(u) d u=1, \int|K(u)| d u<$ $\infty,|u||K(u)| \rightarrow 0$ as $u \rightarrow 0, \sup |K(u)|<\infty, \int K^{2}(u) d u<\infty$ and $\int K^{\prime 2}(u) d u<\infty$. 
(iii) $f_{t}$ is strictly stationary with twice continuously differentiable marginal density $g(f)(>0)$ and is strong-mixing with mixing coefficients $\alpha(j)$ satisfying $\sum_{j=T}^{\infty} \alpha(j)^{1 / 2}=$ $O\left(T^{-1}\right)$, and $\max _{1 \leq t \leq T}\left|f_{t}\right|=O_{p}(\ln T)$.

(iv) $h=$ const. $\times T^{-\beta}$ for $0<\beta<\frac{1}{3}$.

(i) and (ii) are the standard conditions for the nonparametric kernel regression estimators. The last condition in (iii) is from extreme value theory and is expected to hold for a very broad class of distribution. The rate of bandwidth in (iv) allows an optimal rate that minimizes the $\operatorname{MSE}(\beta=1 / 5)$.

Proposition 1. Let $x_{i t}$ and $f_{t}$ be generated from (1) and (2), respectively, and suppose that assumptions $F$ and $K$ are satisfied. Then $\widehat{m}(f)-\widetilde{m}(f)=o_{p}(1 / \sqrt{T h})$ for all $f$.

The results above provide the validity of the two-step estimation applied to the estimated factor. When both $N$ and $T$ tend to infinity, the effect of the estimation error of the factor becomes negligible in the asymptotic distribution of the final estimator, since the variance of the nonparametric kernel estimator $\widehat{m}(f)$ is $O_{p}(1 /(T h))$. While we only provide the proof for $p=1$ case in the Appendix, the result can easily be generalized to the case of higher order models with $p>1$. Furthermore, the result of kernel autoregression in Proposition 1 can be applied to other nonparametric methods, such as neural networks, by replacing the assumptions to the ones suitable for each estimator. 


\section{Simulation Results}

In this section, we study the finite sample performance of the Lyapunov exponent estimator of the common factor for the various combination of $N$ and $T$. In particular, we consider two nonlinear $\mathrm{AR}(1)$ processes to generate $f_{t}$, the logistic map with a positive $\lambda$ and the STAR model with a negative $\lambda .{ }^{1}$ The first model is the logistic map with a system error given by

$$
f_{t}=a f_{t-1}\left(1-f_{t-1}\right)+\sigma_{t} \varepsilon_{t}
$$

where $\varepsilon_{t} \sim$ iid $U(-1 / 2,1 / 2)$ and $\sigma_{t}=\sigma \times \min \left\{a f_{t-1}\left(1-f_{t-1}\right), 1-a f_{t-1}\left(1-f_{t-1}\right)\right\}$. This particular form of conditional heteroskedasticity ensures that the process $f_{t}$ is restricted to the unit interval as long as $0 \leq \sigma \leq 2$. In the case of $\sigma=0$, the dynamics of the logistic map is deterministic chaos when the parameter $a$ is in the range of $3.57<a \leq 4$. In this simulation, we focus on the chaotic case with $a=4$. Note that when $p=1$, the definition of the Lyapunov exponent in equation (3) simplifies to

$$
\lambda \equiv \lim _{M \rightarrow \infty} M^{-1} \sum_{t=1}^{M} \ln \left|m^{\prime}\left(f_{t-1}\right)\right|
$$

where $m^{\prime}(f)$ is the first derivative of the function $m(f)$. When $\sigma=0$, the value of $\lambda$ for equation (6) is positive and is known to be $\ln 2 \approx 0.693$. For moderate $\sigma>0$, equation (6) becomes a stochastic system but its dynamics can be considered as noisy chaos when $\lambda>0$. True $\lambda$ 's of equation (6) for various values of non-zero $\sigma$ are computed using simulation method and provided in Table 1 . It shows that $\lambda$ approaches 0.693 as $\sigma$ becomes smaller.

\footnotetext{
${ }^{1}$ We consider these two nonlinear models because they are often used in economic analysis. For example, a logistic model is used in Day (1982) and a STAR model is used in Michael, Nobay and Peel (1997).
} 
As a second nonlinear model, a smooth transition AR (STAR) model is considered. For a linear $\mathrm{AR}(1)$ model with $m\left(f_{t-1}\right)=\rho f_{t-1}$, the Lyapunov exponent is $\lambda=\ln |\rho|$ since $m^{\prime}(f)=\rho$ for all $f$. Therefore, the stationary condition $|\rho|<1$ corresponds to a negative Lyapunov exponent. For example, in the case of $\rho=0.5, \lambda$ is $-\ln 2 \approx$ -0.693 . In the case of a unit root with $\rho=1, \lambda$ is zero. Therefore, in the linear case with positive $\rho$, the faster speed of adjustment implies smaller $\lambda(\lambda \rightarrow-\infty)$ and the higher persistence implies larger $\lambda(\lambda \rightarrow 0)$. However, in economic analysis, the speed of adjustment is often believed to be faster when deviation from the steady state is larger. The STAR model can be used to incorporate such a nonlinear adjustment. In simulation, we use

$$
f_{t}= \begin{cases}\mu+\rho f_{t-1}+\sigma \varepsilon_{t} & f_{t-1}>c \\ S\left(f_{t-1}\right)+\sigma \varepsilon_{t} & -c \leq f_{t-1} \leq c \\ -\mu+\rho f_{t-1}+\sigma \varepsilon_{t} & f_{t-1}<-c\end{cases}
$$

where $S\left(f_{t-1}\right)=f_{t-1}-f_{t-1}\left\{1-\exp \left(-f_{t-1}^{2}\right)\right\}, \varepsilon_{t} \sim$ iid $N(0,1), \rho=0.5, \mu=S(c)-\rho c$ and $c(>0)$ is a threshold value that satisfies $S^{\prime}(c)=\rho$. The function $S\left(f_{t-1}\right)$ provides a simple STAR structure with the slowest speed of adjustment around the steady state level $f=0$. The linear AR structure outside the $(-c, c)$ band is introduced here to ensure that the speed of adjustment is always positive. In this model, $m^{\prime}(f) \rightarrow 1$ as $f$ approaches 0 and $m^{\prime}(f) \rightarrow \rho=0.5$ as $|f|$ increases. Therefore, we expect that $\lambda$ approaches 0 as $\sigma$ becomes smaller and $\lambda$ approaches $\ln |\rho|=-\ln 2 \approx-0.693$ as $\sigma$ becomes larger. True $\lambda$ 's for various values of $\sigma$ obtained from simulation in Table 1 lie between -0.693 and 0 and thus confirm this conjecture.

We first evaluate the performance of a nonparametric estimator of the Lyapunov exponent in the case of observable $f_{t}$. Such an estimator is not feasible in practice, but can be obtained in the simulation study. In this section, to estimate $m$ function 
in equation (2), we employ a local polynomial estimator which is a type of kernel estimator. Note that the Nadaraya-Watson estimator considered in the previous section is a local polynomial estimator of order zero (local constant estimator). Here we employ the local polynomial estimator of order two (local quadratic estimator) since this choice of order is known to be most appropriate for the first derivative estimation required for the Lyapunov exponent computation (See Fan and Gijbels, $1996)^{2}$

Table 1 reports the results for $T=50,100,200,400$ and 800 for both the logistic map and the STAR model based on 1,000 iteration. ${ }^{3}$ The set of values of $\sigma$ under consideration is $\sigma \in\{0.00,0.05,0.10,0.20,0.50\}$ for the logistic map and $\sigma \in\{0.05$, $0.10,0.20,0.50,1.00\}$ for the STAR model. For the logistic map with positive true $\lambda$, the Lyapunov exponent estimator performs surprisingly well for all the cases. The mean of the estimators shows that small sample bias is almost negligible even with $T=50$. Decreasing standard deviation with increasing $T$ confirms the consistency of the estimator. In contrast, the small sample bias seems to be present for the STAR model with negative true $\lambda$. This bias, however, diminishes quickly as sample size grows. The standard deviation is somewhat larger than that of logistic map case for the corresponding $T$, but is decreasing with $T$ confirming the adequacy of asymptotic theory. The difference between mean and median implies some skewness to the left for small $T$ but it disappears for large $T$. Because we cannot expect the feasible estimator based on the estimated factor to perform better than the infeasible estimator, this

\footnotetext{
${ }^{2}$ Since the nonlinear estimation is not invariant to scale, we always normalize the factors in the $(0,1)$ unit interval before running a nonparametric regression for this paper.

${ }^{3}$ We use standard normal density as a kernel function (Gaussian kernel) with several different choices of bandwidth $h$. In the table, the results based on $h=0.3 \times$ range are reported.
} 
result serves as a benchmark in the following analysis.

Let us now consider the Lyapunov exponent estimation applied to the factor estimated using principal components. Individual series $x_{i t}$ is generated from equation (1) with $e_{i t}$ and $b_{i}$ being iid $N(0,1)$. The common factor $f_{t}$ generated from both the logistic map and the STAR model is normalized to have unit variance before generating $x_{i t}$ so that the common component $b_{i} f_{t}$ and the idiosyncratic component $e_{i t}$ each has the same contribution to the variance of $x_{i t}$.

We evaluate the performance of our estimator for all the combinations of $N=25$, 50, 100, 200, and 400 and $T=100,200$, and 400. Tables 2 to 4 report the results for $T=100,200$, and 400, respectively, using various values of $N$. For each table, the results from the infeasible estimator for corresponding $T$ are also reported for the purpose of comparison with our proposed estimator. For each table with fixed $T$, the estimator applied to the principal components of $x_{i t}$ approaches the infeasible estimator as $N$ grows. This evidence combined with the results from Table 1 suggests convergence of the estimator predicted by the asymptotic theory under $N, T \rightarrow \infty$. The bias from small $N$ turns out to be negative for both the logistic map and the STAR model. In addition, the skewness with small $N$ can be now seen in the case of the logistic map, which was not present in the infeasible estimators with small $T$. This asymmetry in distribution, however, is not observed when $N$ becomes as large as 100. In contrast, some skewness is present for large $N$ in the case of the STAR model with small $T$ because of the skewness in the infeasible estimator.

Overall, the asymptotic approximation seems to work well for many combinations of $N$ and $T$ typically available in practice. Most importantly, the small standard deviation in the simulation implies the successful detection of correct sign of $\lambda$ for 
both the logistic map and the STAR model despite the presence of some finite sample bias from small $N$. Since the positive $\lambda$ is the key to distinguishing the chaotic process from the stable system, we expect that conducting the inference regarding the sign of $\lambda$ of the principal components will work well in the next empirical section.

\section{Empirical Results}

\subsection{Testing for Neglected Nonlinearity in Principal Compo- nents}

In this section, we investigate the nonlinear stability of the common factors extracted from the 159 monthly macroeconomic variables from the U.S. We use the same data source (and transformations) employed in Stock and Watson (2002a) in their analysis of macroeconomic forecasting using estimated factors from a large number of predictors (diffusion index forecast), but we focus only on balanced panels. The series in a list provided in Appendix B of Stock and Watson (2002a) are divided into 14 categories, each of which represents an important facet of the macroeconomic activities (e.g., production, consumption, employment, inflation, interest rates). The sample period is from 1959:3 to 1998:12, giving a maximum number of time series observations $T=478$. All the series are standardized to have sample mean zero and unit sample variance since principal components are not scale-invariant.

The estimated series of $\widetilde{f}_{t}$ based on the first principal component of the full balanced panel series $x_{i t}$ with $N=159$ is shown in Figure 1. In the figure, $\widetilde{f}_{t}$ is rescaled to have the same drift and variance as the (logarithm of) industrial production. ${ }^{4}$ The comparison of $\widetilde{f}_{t}$ with the NBER recessionary episodes, shown in the shaded area,

\footnotetext{
${ }^{4}$ Therefore, the series in the figure is equivalent to the cummulated sum of the first principal component rather than the original series.
} 
shows the clear procyclical property of the common factor. The asymptotic theory predicts the $\tilde{f}_{t}$ to be a consistent estimator of $f_{t}$ as $N \rightarrow \infty$. To investigate this prediction using the data, we take first five and two series from each category in Appendix B of Stock and Watson (2002a) and compute $\widetilde{f}_{t}$ based on two subsamples with $N=63$ and 27. Two additional series of $\widetilde{f}_{t}$ are also shown in the same figure. Consistent with the theory, it shows a larger difference in $\widetilde{f}_{t}$ between $N=27$ and $N=159$ compared to the difference between $N=63$ and $N=159$.

As a preliminary analysis, we first investigate the nonlinearity in the factor dynamics in equation (2). In applications, a linear structure is often assumed in equation (2) to simplify the analysis (e.g., Stock and Watson, 1989). Since the chaotic dynamics with positive $\lambda$ is only possible with nonlinear dynamics, we employ several tests for the null hypothesis of linearity (or neglected nonlinearity) that has a power against a wide range of nonlinear alternatives. When $p=1$, our testing hypothesis is $m\left(f_{t-1}\right)=\rho f_{t-1}$, or $E\left[u_{t} \mid f_{t-1}\right]=0$, where $u_{t}=f_{t}-\rho f_{t-1}$. This hypothesis can be rewritten as the unconditional moment restriction of the form $E\left[\Psi\left(f_{t-1}\right) u_{t}\right]=0$ with any vector of measurable functions $\Psi\left(f_{t-1}\right)$; thus a number of tests can be constructed with the different choice of $\Psi\left(f_{t-1}\right)$.

Ramsey's (1969) regression specification error test (RESET), which is one of the most well-known tests in the specification testing literature, uses a $s \times 1$ vector of polynomial functions of fitted value from linear regression, $\Psi\left(f_{t-1}\right)=\left(\left(\widehat{\rho} f_{t-1}\right)^{2}, \ldots,\left(\widehat{\rho} f_{t-1}\right)^{s}\right)^{\prime}$. The test statistic is defined as RESET $=T\left(\sum_{t=1}^{T} \widehat{u}_{t}^{2}-\sum_{t=1}^{T} \widehat{v}_{t}^{2}\right) / \sum_{t=1}^{T} \widehat{u}_{t}^{2}$, where $\widehat{u}_{t}=f_{t}-\widehat{\rho} f_{t-1}$ and $\widehat{v}_{t}$ are the residuals from the regression of $\widehat{u}_{t}$ on auxiliary regressors $\Psi\left(f_{t-1}\right)$ (and $\left.f_{t-1}\right)$, and asymptotically follows $\chi^{2}$ distribution with $s$ degree of freedom. 
For White's (1989) neural network test, $\Psi\left(f_{t-1}\right)=\left(\psi\left(\gamma_{1} f_{t-1}\right), \ldots, \psi\left(\gamma_{q} f_{t-1}\right)\right)^{\prime}$ is a $q \times 1$ vector of logistic activation functions $\psi$ with the coefficients $\gamma_{j}$ 's being randomly drawn independent of $f_{t-1}$. The test statistic (NN) can be similarly constructed by using auxiliary regressors and its limit distribution is $\chi^{2}$ distribution with $q$ degree of freedom.

One drawback of the White's neural network test is the unidentifiability of $\gamma_{j}$ 's under the null hypothesis. Instead of using random $\gamma_{j}$ 's, Teräsvirta, Lin, and Granger (1993) replaced the activation functions with their Volterra expansion up to the third order under the null. This LM type neural network test (NN-LM) can be constructed by using auxiliary regressors based on quadratic and cubic terms from the Volterra expansion of the nonlinear AR model $\left(\Psi\left(f_{t-1}\right)=\left(\left(f_{t-1}\right)^{2},\left(f_{t-1}\right)^{3}\right)^{\prime}\right.$ for AR(1) case). The test statistic asymptotically follows $\chi^{2}$ distribution with $p(p+1) / 2+p(p+1)(p+$ 2)/6 degree of freedom where $p$ is lag order of AR model.

The last test we consider is the kernel-based consistent specification test for AR models proposed by Fan and $\mathrm{Li}(1997)$. It utilizes the $\Psi\left(f_{t-1}\right)=E\left(u_{t} \mid f_{t-1}\right) g\left(f_{t-1}\right)$ where $g\left(f_{t-1}\right)$ is a density function of $f_{t-1}$. The test statistic (KERNEL) is based on kernel estimator of $E\left[\Psi\left(f_{t-1}\right) u_{t}\right]$ and follows asymptotically normal with an appropriate standardization.

We apply these four tests to $\widetilde{f}_{t}$, which is estimated by the principal components method since $f_{t}$ is not available. In addition to the first principal component, we apply the same tests to the $k$-th principal component for $k=2$ to 6 . Table 5 reports the results of all four tests applied to each principal component for $p=1$ to 4 . For RESET, the results based on $s=4$ are reported. For NN, we use three (excluding the first) principal components of $\Psi\left(f_{t-1}\right)$ with $q=10$ to avoid collinearity of $f_{t-1}$ and 
$\Psi\left(f_{t-1}\right)$. Improved Bonferroni procedures from five draws is then used to construct $p$ values (see Lee, White, and Granger, 1993, for this procedure in detail). The RESET, NN, and NN-LM tests reject the linear hypothesis of the common factor for many cases at the conventional significance level. In contrast, based on the KERNEL test, the same hypothesis is not rejected for all cases. One possible reason of this mixed outcome is the difference in the power among the specification tests. ${ }^{5}$ However, since at least three tests provided evidence against linearity, we proceed to the estimation and test of the Lyapunov exponent of the common factor using the nonparametric regression method.

\subsection{Lyapunov Exponents of Principal Components}

Here we compute the Lyapunov exponent, $\lambda$, of the common factor obtained from the principal components. As an estimation method of the nonlinear AR model of equation (2), we employ a kernel-based nonparametric method - the local polynomial estimator of order two - the method used in the simulation section. In addition to the kernel-based method, we also consider the neural networks as an alternative method for estimating the nonlinear function. The first and latter methods can be considered as the local and global nonparametric methods, respectively, and thus reporting both results should provide useful information regarding the robustness of our approach to the choice of the estimator. ${ }^{6}$ The stability of the nonlinear system can be directly investigated by conducting a hypothesis test regarding the sign of $\lambda$ using the standard error of the estimator of $\lambda$ (see Shintani and Linton, 2004, for this procedure in detail).

\footnotetext{
${ }^{5}$ Lee's (2001) simulation study compared the performance of NN and KERNEL and reported that KERNEL was less powerful than NN unless the bootstrapped critical value was used.

${ }^{6}$ These two methods are also employed in Shintani and Linton (2003) in their study of nonlinear stability in the international output series.
} 
Table 6 shows the Lyapunov exponent estimates of the principal components of 159 series based on the nonlinear AR model with $p=1$ to 4 . In addition to $\lambda$ of the first principal component, those of the $k$-th principal component from $k=2$ to 6 are also reported. Both full sample estimates $(M=T)$ and block estimates $(M<T)$ are presented. ${ }^{7}$ For the kernel-based estimation, Gaussian kernel function, $K$, is used with the bandwidth $h=0.2 \times$ range. For the neural network estimation, the logistic activation function, $\psi$, is used with the number of hidden units $q=4 .^{8}$ For all cases, the Lyapunov exponents of the first principal component are significantly negative, implying evidence against a chaotic explanation of business cycles. This empirical finding is robust to the choice of nonparametric methods - kernel regression and neural networks.

The second to sixth principal components also provide evidence against chaos while the second principal component has a somewhat larger exponent in comparison with the other principal components.

These results are consistent with the former studies that applied the nonparametric Lyapunov exponent test to a single time series. It is worth pointing out that we are considering a system of a large number of variables and still obtaining evidence of nonlinear stability.

\footnotetext{
${ }^{7}$ For block estimation, we use $M=70$ and report the median based on six blocks. Here, $M$ is approximately $8 \times(T / \ln T)^{1 / 2}$.

${ }^{8}$ To solve the minimization problem required for the neural network estimation, the criterion function is modified to have the weight decay identical to the one employed in Franses and van Dijk (2000).
} 


\subsection{Nonlinear Impulse Response Function of Principal Com- ponents}

The negative Lyapunov exponents can be considered an empirical justification of the impulse response analysis that is commonly used among macroeconomists since it requires the assumption of the exogenous shocks and a stable steady state in the system.

For the nonlinear system, the nonlinear impulse response analysis was proposed by Gallant, Rossi, and Tauchen (1993), and Potter (2000). Here we compute the nonlinear impulse response function of the common factors based on the nonparametric method. One of the most frequently used definitions of the $n$-period ahead nonlinear impulse response is

$$
\begin{aligned}
I R_{n}(\delta) & =E\left(f_{t+n-1} \mid \mathbf{f}_{t-1}=\mathbf{f}^{*}\right)-E\left(f_{t+n-1} \mid \mathbf{f}_{t-1}=\mathbf{f}\right) \\
& =m_{n}\left(\mathbf{f}^{*}\right)-m_{n}(\mathbf{f})
\end{aligned}
$$

where $\mathbf{f}_{t-1}=\left(f_{t-1}, \ldots, f_{t-p}\right), \mathbf{f}^{*}=\left(\mu_{f}+\delta, \mu_{f}, \ldots, \mu_{f}\right)$ and $\mathbf{f}=\left(\mu_{f}, \mu_{f}, \ldots, \mu_{f}\right)$. Unlike the linear impulse response function, the nonlinear impulse response function is known to depend on the size of shock $\delta$ and the initial condition $\mu_{f}$. Note that the mean $\mu_{f}$ is identical to the steady state level in the absence of shocks for a globally stable system with negative $\lambda$. In contrast, in the case of chaos, $\mu_{f}$ cannot be considered as the steady state. In addition, a small difference in $\delta$ results in completely different shapes of the impulse responses. Therefore, the nonlinear impulse response analysis is inappropriate for a system with positive $\lambda$ since it cannot represent the typical shock propagation process in the system.

The nonlinear impulse response function can be obtained by estimating the $n$ - 
period ahead conditional mean function $m_{n}\left(f_{t-1}, \ldots, f_{t-p}\right)$ using the same nonparametric method used in the estimation of 1-period ahead conditional mean function $m\left(f_{t-1}, \ldots, f_{t-p}\right)=m_{1}\left(f_{t-1}, \ldots, f_{t-p}\right)$ in equation (2). As in the 1-period ahead case, nonparametric regression $\widehat{m}_{n}(\mathbf{f})$ based on $f_{t}$ is not feasible and $\widetilde{m}_{n}(\mathbf{f})$ based on $\widetilde{f}_{t}$ need to be employed.

Figures $2 \mathrm{~A}$ to $2 \mathrm{D}$ show the nonlinear impulse response function for the case of $p=1$ to 4 , respectively. For the size of the shock, we use $\delta \in\{+2 \sigma,+\sigma,-\sigma$, $-2 \sigma\}$ where $\sigma$ is the standard deviation of the shocks estimated using the residuals of the nonparametric regression of equation (2). The linear impulse responses from the linear AR models are also shown in the figures for comparison. The shapes of impulse responses in figures show a clear difference between the linear and nonlinear cases.

Recall that the larger Lyapunov exponent was obtained for the second principal component $(k=2)$ compared to the first principal component $(k=1)$ in the previous section. The nonlinear impulse responses of the second principal component are shown in Figures 3A to 3D for the case of $p=1$ to 4 , respectively. Comparisons with Figures $2 \mathrm{~A}$ to $2 \mathrm{D}$ reveal a much higher persistence of common shocks in the second factor, which is consistent with a less stable result from the Lyapunov exponent. Halflives computed from these impulse responses can be used to measure the persistence in common shocks. For example, when $p=2$, half-lives from the linear impulse responses are 1.20 months for $k=1$ and 3.54 months for $k=2$. When nonlinear impulse responses are used, the half-lives of the $+2 \sigma,+\sigma,-\sigma$, and $-2 \sigma$ shocks in the first principal component $(k=1)$ are $0.87,0.93,0.97$, and 1.00 months, respectively. In contrast, the half-lives of corresponding shocks in the second principal component 
$(k=2)$ are 5.05, 5.06, 5.27, and 5.32 months, respectively. According to Stock and Watson (2002a, p.153), the first factor loads primarily on output and employment and the second factor, on interest rate spreads, unemployment rates, and capacity utilization rates. While identification of each shock in two factors is not our intention here, the results of our nonlinear analysis seem to provide some useful information regarding the characteristics of the source of business cycles.

\section{Conclusion}

The largest Lyapunov exponent is a useful nonlinear stability measure in distinguishing chaos from an exogenous shock-driven aggregate fluctuation in an economy. In practice, this measure is nonparametrically estimated from a single economic time series. Such an approach, however, cannot be applied to test the nonlinear stability of a large system of equations because of the dimensionality problem of the nonparametric methods. To circumvent this problem, we proposed computing the nonlinear stability measure of the common component of multiple time series under the framework of a dynamic factor model.

We employed a method of principal components to estimate the unobservable common factors since the method is known to provide a consistent estimator when a large panel data is available. Furthermore, the nonparametric regression based on the estimated factor was shown to be a valid approach to investigating the nonlinear dynamic factor structure. The simulation evidence also suggested that our procedure worked well with a sample size typically available in practice.

The empirical evidence from a large U.S. panel data suggested the possibility of nonlinearity in the system while it excluded the class of nonlinearity that could 
generate endogenous fluctuation or chaos. This result was consistent with the former studies that applied a nonparametric Lyapunov exponent test to a single time series (e.g., Shintani and Linton, 2003). Therefore, we now have evidence on nonlinear stability not only within a univariate or a small system of equations but also within a system of a large number of variables. 


\section{Appendix A: Proofs}

Lemma A.1. Suppose assumptions $F$ and $K$ are satisfied. Then,

$$
\begin{aligned}
& \text { (i) } \frac{1}{\sqrt{T h}} \sum_{t=1}^{T}\left(\widetilde{f}_{t}-H f_{t}\right) K\left(\frac{f_{t-1}-f}{h}\right)=o_{p}(1), \\
& \text { (ii) } \frac{1}{\sqrt{T h^{3}}} \sum_{t=1}^{T}\left(\widetilde{f}_{t-1}-H f_{t-1}\right) K^{\prime}\left(\frac{f_{t-1}-f}{h}\right)=o_{p}(1), \\
& \text { (iii) } \frac{1}{\sqrt{T h^{3}}} \sum_{t=1}^{T}\left(\widetilde{f}_{t-1}-H f_{t-1}\right) f_{t} K^{\prime}\left(\frac{f_{t-1}-f}{h}\right)=o_{p}(1), \text { and } \\
& \text { (iv) } \frac{1}{\sqrt{T h^{3}}} \sum_{t=1}^{T}\left(\widetilde{f}_{t}-H f_{t}\right)\left(\widetilde{f}_{t-1}-H f_{t-1}\right) K^{\prime}\left(\frac{f_{t-1}-f}{h}\right)=o_{p}(1)
\end{aligned}
$$

where $H=v^{-1}\left(\widetilde{F}^{\prime} F / T\right)\left(B^{\prime} B / N\right), \widetilde{F}=\left[\widetilde{f}_{1}, \cdots, \widetilde{f}_{T}\right]^{\prime}$ and $v$ is the largest eigenvalue of $X X^{\prime} / T N$.

\section{Proof of Lemma A.1.}

From Proposition 2 of Bai $(2003), \max _{1 \leq t \leq T}\left|\widetilde{f}_{t}-H f_{t}\right|=O_{p}\left(T^{-1 / 2}\right)$ given $N / T^{2} \rightarrow$ $\infty$. The result for Lemma A.1(i) follows from

$$
\begin{aligned}
& \frac{1}{\sqrt{T h}} \sum_{t=1}^{T}\left(\widetilde{f}_{t}-H f_{t}\right) K\left(\frac{f_{t-1}-f}{h}\right) \\
\leq & \sqrt{T h}\left(\frac{1}{T h} \sum_{t=1}^{T} K\left(\frac{f_{t-1}-f}{h}\right)\right) \max _{1 \leq t \leq T}\left|\widetilde{f}_{t}-H f_{t}\right| \\
= & O_{p}(\sqrt{T h}) \cdot O_{p}(1) \cdot O_{p}\left(T^{-1 / 2}\right)=O_{p}\left(T^{-\beta / 2}\right) .
\end{aligned}
$$

Similarly, for (ii),

$$
\begin{aligned}
& \frac{1}{\sqrt{T h^{3}}} \sum_{t=1}^{T}\left(\widetilde{f}_{t-1}-H f_{t-1}\right) K^{\prime}\left(\frac{f_{t-1}-f}{h}\right) \\
\leq & \sqrt{T h}\left(\frac{1}{T h^{2}} \sum_{t=1}^{T} K^{\prime}\left(\frac{f_{t-1}-f}{h}\right)\right) \max _{1 \leq t \leq T}\left|\widetilde{f}_{t}-H f_{t}\right| \\
= & O_{p}(\sqrt{T h}) \cdot O_{p}(1) \cdot O_{p}\left(T^{-1 / 2}\right)=O_{p}\left(T^{-\beta / 2}\right) .
\end{aligned}
$$

The results for (iii) and (iv) follow from

$$
\begin{aligned}
& \frac{1}{\sqrt{T h^{3}}} \sum_{t=1}^{T}\left(\widetilde{f}_{t-1}-H f_{t-1}\right) f_{t} K^{\prime}\left(\frac{f_{t-1}-f}{h}\right) \\
\leq & \sqrt{T h}\left(\frac{1}{T h^{2}} \sum_{t=1}^{T} K^{\prime}\left(\frac{f_{t-1}-f}{h}\right)\right) \max _{1 \leq t \leq T}\left|\widetilde{f}_{t}-H f_{t}\right| \max _{1 \leq t \leq T}\left|f_{t}\right| \\
= & O_{p}(\sqrt{T h}) \cdot O_{p}(1) \cdot O_{p}\left(T^{-1 / 2}\right) \cdot O_{p}(\ln T)=O_{p}\left(\frac{\ln T}{T^{\beta / 2}}\right) .
\end{aligned}
$$

and 


$$
\begin{aligned}
& \frac{1}{\sqrt{T h^{3}}} \sum_{t=1}^{T}\left(\widetilde{f}_{t}-H f_{t}\right)\left(\widetilde{f}_{t-1}-H f_{t-1}\right) K^{\prime}\left(\frac{f_{t-1}-f}{h}\right) \\
\leq & \sqrt{T h}\left(\frac{1}{T h^{2}} \sum_{t=1}^{T} K^{\prime}\left(\frac{f_{t-1}-f}{h}\right)\right)\left(\max _{1 \leq t \leq T}\left|\widetilde{f}_{t}-H f_{t}\right|\right)^{2} \\
= & O_{p}(\sqrt{T h}) \cdot O_{p}(1) \cdot O_{p}\left(T^{-1}\right)=O_{p}\left(T^{-(\beta+1) / 2}\right) .
\end{aligned}
$$

Proof of Proposition 1.

$$
\begin{aligned}
& \sqrt{T h}(\widetilde{m}(f)-\widehat{m}(f)) \\
= & {\left[\frac{1}{\sqrt{T h}} \sum_{t=1}^{T} \widetilde{f}_{t} K\left(\frac{\widetilde{f}_{t-1}-f}{h}\right)\right] / \widetilde{g}(f)-\left[\frac{1}{\sqrt{T h}} \sum_{t=1}^{T} f_{t} K\left(\frac{f_{t-1}-f}{h}\right)\right] / \widehat{g}(f) } \\
= & \left\{\frac{1}{\sqrt{T h}} \sum_{t=1}^{T} \widetilde{f}_{t} K\left(\frac{\widetilde{f}_{t-1}-f}{h}\right)-\frac{1}{\sqrt{T h}} \sum_{t=1}^{T} f_{t} K\left(\frac{f_{t-1}-f}{h}\right)\right\} / \widetilde{g}(f) \\
& +\left(\frac{1}{\widetilde{g}(f)}-\frac{1}{\widehat{g}(f)}\right)\left[\frac{1}{\sqrt{T h}} \sum_{t=1}^{T} f_{t} K\left(\frac{f_{t-1}-f}{h}\right)\right] \\
= & \left\{\frac{1}{\sqrt{T h}} \sum_{t=1}^{T} \widetilde{f}_{t} K\left(\frac{f_{t-1}-f}{h}\right)-\frac{1}{\sqrt{T h}} \sum_{t=1}^{T} f_{t} K\left(\frac{f_{t-1}-f}{h}\right)\right\} / \widetilde{g}(f) \\
& +\left\{\frac{1}{\sqrt{T h}} \sum_{t=1}^{T} \widetilde{f}_{t} K\left(\frac{\widetilde{f}_{t-1}-f}{h}\right)-\frac{1}{\sqrt{T h}} \sum_{t=1}^{T} \widetilde{f}_{t} K\left(\frac{f_{t-1}-f}{h}\right)\right\} / \widetilde{g}(f) \\
& +\sqrt{T h}\left(\frac{1}{\widetilde{g}(f)}-\frac{1}{\widehat{g}(f)}\right)\left[\frac{1}{T h} \sum_{t=1}^{T} f_{t} K\left(\frac{f_{t-1}-f}{h}\right)\right] \\
= & a_{T}^{*}+b_{T}^{*}+c_{T}^{*}, \text { say, }
\end{aligned}
$$

where

$$
\widehat{g}(f)=\frac{1}{T h} \sum_{t=1}^{T} K\left(\frac{f_{t-1}-f}{h}\right) \quad \text { and } \quad \widetilde{g}(f)=\frac{1}{T h} \sum_{t=1}^{T} K\left(\frac{\widetilde{f}_{t-1}-f}{h}\right) .
$$

For the numerator of $a_{T}^{*}$, we have

$$
\begin{aligned}
& \frac{1}{\sqrt{T h}} \sum_{t=1}^{T}\left(\widetilde{f}_{t}-f_{t}\right) K\left(\frac{f_{t-1}-f}{h}\right) \\
= & \frac{1}{\sqrt{T h}} \sum_{t=1}^{T}\left(\widetilde{f}_{t}-H f_{t}\right) K\left(\frac{f_{t-1}-f}{h}\right)+\frac{1}{\sqrt{T h}}(H-1) \sum_{t=1}^{T} f_{t} K\left(\frac{f_{t-1}-f}{h}\right) \\
= & o_{p}(1)+O_{p}(\sqrt{h})=o_{p}(1) .
\end{aligned}
$$


The convergence of the first term follows from Lemma A.1(i). For the second term, we used

$$
\begin{aligned}
& \sqrt{T h}(H-1) \frac{1}{T h} \sum_{t=1}^{T} f_{t} K\left(\frac{f_{t-1}-f}{h}\right) \\
= & O_{p}(\sqrt{T h}) O_{p}\left(T^{-1 / 2}\right)=O_{p}(\sqrt{h})
\end{aligned}
$$

where $H-1=O_{p}\left(\min (\sqrt{T}, \sqrt{N})^{-1}\right)=O_{p}\left(T^{-1 / 2}\right)$ follows from Assumption $\mathrm{F}(\mathrm{i})$ and (ii). For the numerator of $b_{T}^{*}$, from a mean value expansion, it can be rewritten as

$\frac{1}{\sqrt{T h}} \sum_{t=1}^{T} \widetilde{f}_{t} K^{\prime}\left(\frac{f_{t-1}-f}{h}\right)\left(\frac{\widetilde{f}_{t-1}-f_{t-1}}{h}\right)+\frac{1}{\sqrt{T h}} \sum_{t=1}^{T} \widetilde{f}_{t} K^{\prime \prime}\left(\frac{f_{t-1}^{*}-f}{h}\right)\left(\frac{\widetilde{f}_{t-1}-f_{t-1}}{h}\right)^{2}$

where $f_{t-1}^{*}$ lies between $\widetilde{f}_{t-1}$ and $f_{t-1}$. Since the first term dominates the second term, it suffices to show the convergence of the first term.

$$
\begin{aligned}
& \frac{1}{\sqrt{T h^{3}}} \sum_{t=1}^{T} \widetilde{f}_{t} K^{\prime}\left(\frac{f_{t-1}-f}{h}\right)\left(\widetilde{f}_{t-1}-f_{t-1}\right) \\
= & \frac{1}{\sqrt{T h^{3}}} \sum_{t=1}^{T} \widetilde{f}_{t}\left(\widetilde{f}_{t-1}-H f_{t-1}\right) K^{\prime}\left(\frac{f_{t-1}-f}{h}\right)+\frac{1}{\sqrt{T h^{3}}}(H-1) \sum_{t=1}^{T} \widetilde{f}_{t} f_{t-1} K^{\prime}\left(\frac{f_{t-1}-f}{h}\right) \\
= & \frac{1}{\sqrt{T h^{3}}} \sum_{t=1}^{T}\left(\widetilde{f}_{t}-H f_{t}\right)\left(\widetilde{f}_{t-1}-H f_{t-1}\right) K^{\prime}\left(\frac{f_{t-1}-f}{h}\right) \\
& +H \frac{1}{\sqrt{T h^{3}}} \sum_{t=1}^{T}\left(\widetilde{f}_{t-1}-H f_{t-1}\right) f_{t-1} K^{\prime}\left(\frac{f_{t-1}-f}{h}\right) \\
& +(H-1) \frac{1}{\sqrt{T h^{3}}} \sum_{t=1}^{T}\left(\widetilde{f}_{t}-H f_{t}\right) f_{t-1} K^{\prime}\left(\frac{f_{t-1}-f}{h}\right) \\
& +\sqrt{T h} H(H-1) \frac{1}{T h^{2}} \sum_{t=1}^{T} f_{t} f_{t-1} K^{\prime}\left(\frac{f_{t-1}-f}{h}\right) \\
= & o_{p}(1) .
\end{aligned}
$$

For the first to third terms, convergence follows from Lemma A.1(iii) and (iv). Similarly, from a mean value expansion, we have

$$
\begin{aligned}
& \sqrt{T h}(\widetilde{g}(f)-\widehat{g}(f)) \\
= & \frac{1}{\sqrt{T h}} \sum_{t=1}^{T} K^{\prime}\left(\frac{f_{t-1}-f}{h}\right)\left(\frac{\widetilde{f}_{t-1}-f_{t-1}}{h}\right)+\frac{1}{\sqrt{T h}} \sum_{t=1}^{T} K^{\prime \prime}\left(\frac{f_{t-1}^{*}-f}{h}\right)\left(\frac{\widetilde{f}_{t-1}-f_{t-1}}{h}\right)^{2}
\end{aligned}
$$

with the dominant first term being $o_{p}(1)$ from Lemma A.1(ii). Since $\widehat{g}(f)^{-1}=O_{p}(1)$, we have $\sqrt{T h}\left(\widetilde{g}(f)^{-1}-\widehat{g}(f)^{-1}\right)=o_{p}(1)$ and $c_{T}^{*}=o_{p}(1)$. Furthermore, $\widetilde{g}(f)^{-1}=O_{p}(1)$ implies both $a_{T}^{*}$ and $b_{T}^{*}$ to be $o_{p}(1)$ since the numerators are shown to be $o_{p}(1)$. 


\section{References}

Abhyankar, A., Copeland, L.S., Wong, W., 1997. Uncovering nonlinear structure in real-time stock-market indexes: The S\&P 500, the DAX, the Nikkei 225, and the FTSE-100. Journal of Business and Economic Statistics 15, 1-14.

Andrews, D.W.K., 1991. Heteroskedasticity and autocorrelation consistent covariance matrix estimation. Econometrica 59, 817-858.

Andrews, D.W.K., 1995. Nonparametric kernel estimation for semiparametric models. Econometric Theory 11, 560-596.

Bai, J., 2003. Inferential theory for factor models of large dimensions. Econometrica $71,135-171$.

Barnett, W.A., Gallant, A.R., Hinich, M.J., Jungeilges, J.A., Kaplan, D.T., Jensen, M.J., 1995. Robustness of nonlinearity and chaos tests to measurement error, inference method, and sample size. Journal of Economic Behavior and Organization 27, 301-320.

Bask, M., de Luna, X., 2002. Characterizing the degree of stability of non-linear dynamic models. Studies in Nonlinear Dynamics and Econometrics 6, Article 3.

Brock, W.A., Hommes, C.H., 1998. Heterogeneous beliefs and routes to chaos in a simple asset pricing model. Journal of Economic Dynamics and Control 22, 1235- 1274 .

Chauvet, M., 1998. An econometric characterization of business cycle dynamics with factor structure and regime switching. International Economic Review 39, 969-996.

Day, R. H., 1982. Irregular growth cycles. American Economic Review 72, 406-414.

Dechert, W.D., Gençay, R., 1992. Lyapunov exponents as a nonparametric diagnostic for stability analysis. Journal of Applied Econometrics 7, S41-S60.

Diebold, F.X., 2003. 'Big data' dynamic factor models for macroeconomic measurement and forecasting. in: Dewatripont, M., Hansen, L.P., Turnovsky, S. (Eds.), Advances in Economics and Econometrics: Theory and Applications, Eighth World Congress of the Econometric Society. Cambridge University Press, Cambridge, pp. 115-122.

Eckmann, J.-P., Kamphorst, S.O., Ruelle, D., Ciliberto, S., 1986. Liapunov exponents from time series. Physical Review A 34, 4971-4979. 
Eckmann, J.-P., Ruelle, D., 1985. Ergodic theory of chaos and strange attractors. Reviews of Modern Physics 57, 617-656.

Fan, J., Gijbels, 1996. Local Polynomial Modelling and Its Applications. Chapman and Hall, London.

Fan, Y., Li, Q., 1997. A consistent nonparametric test for linearity of AR(p) models. Economics Letters 55, 53-59.

Franses, P.H., van Dijk, D., 2000. Nonlinear Time Series Models in Empirical Finance. Cambridge University Press, Cambridge.

Gallant, A.R., Rossi, P.E., Tauchen, G., 1993. Nonlinear dynamic structures. Econometrica 61, 871-907.

Gençay, R., Dechert, W.D., 1992. An algorithm for the $\mathrm{n}$ Lyapunov exponents of an n-dimensional unknown dynamical system. Physica D 59, 142-157.

Geweke, J., 1977. The dynamic factor analysis of economic time-series models. in: Aigner, D.J., Goldberger, A.S. (Eds.), Latent Variable in Socioeconomic Models, North-Holland, Amsterdam, pp. 365-387.

Kim, C.-J., Nelson, C.R., 1998. Business cycle turning points, a new coincident index, and tests of duration dependence based on a dynamic factor model with regime-switching. Review of Economics and Statistics 80, 188-201.

Lee, T.-H., 2001. Neural network test and nonparametric kernel test for neglected nonlinearity in regression models. Studies in Nonlinear Dynamics and Econometrics 4, 169-182.

Lee, T.-H., White, H., Granger, C.W.J., 1993. Testing for neglected nonlinearity in time series models: A comparison of neural network methods and alternative tests. Journal of Econometrics 56, 269-290.

McCaffrey, D.F., Ellner, S., Gallant, A.R., Nychka, D.W., 1992. Estimating the Lyapunov exponent of a chaotic system with nonparametric regression. Journal of the American Statistical Association 87, 682-695.

Michael, P., Nobay, A.R., Peel, D.A., 1997. Transaction costs and nonlinear adjustments in real exchange rates: An empirical investigation. Journal of Political Economy 105, 862-879.

Nychka, D., Ellner, S., Gallant, A.R., McCaffrey, D., 1992. Finding chaos in noisy system. Journal of the Royal Statistical Society B 54, 399-426. 
Potter, S.M., 2000. Nonlinear impulse response functions. Journal of Economics Dynamics and Control 24, 1425-1446.

Ramsey, J.B., 1969. Tests for specification errors in classical linear least-squares regression analysis. Journal of the Royal Statistical Society B 31, 350-371.

Sargent, T.J., Sims, C.A., 1977. Business cycle modeling without pretending to have too much a priori economic theory. in: Sims, C.A. (Ed.), New Methods of Business Cycle Research. Federal Reserve Bank of Minneapolis, Minneapolis, pp. $45-109$.

Serletis, A., 1995. Random walks, breaking trend functions, and the chaotic structure of the velocity of money. Journal of Business and Economic Statistics 13, 453-458.

Shintani, M., 2004. Nonlinear forecasting analysis using diffusion indexes: An application to Japan. Journal of Money, Credit, and Banking, forthcoming.

Shintani, M., Linton, O., 2003. Is there chaos in the world economy? A nonparametric test using consistent standard errors, International Economic Review 44, 331-358.

Shintani, M., Linton, O., 2004. Nonparametric neural network estimation of Lyapunov exponents and a direct test for chaos, Journal of Econometrics 120, 1-33.

Stock, J.H., Watson, M.W., 1989. New indexes of coincident and leading economic indicators. in: Blanchard, O., Fischer, S. (Eds.), NBER Macroeconomics Annual. MIT Press, Cambridge, pp. 351-394.

Stock, J.H., Watson, M.W., 2002a. Macroeconomic forecasting using diffusion indexes. Journal of Business and Economic Statistics 20, 147-162.

Stock, J.H., Watson, M.W., 2002b. Forecasting using principal components from a large number of predictors. Journal of the American Statistical Association 97, 1167-1179.

Teräsvirta, T., Lin, C.-F., Granger, C.W.J., 1993. Power of the neural network linearity test. Journal of Time Series Analysis 14, 209-220.

Whang, Y.-J., Linton, O., 1999. The asymptotic distribution of nonparametric estimates of the Lyapunov exponent for stochastic time series. Journal of Econometrics 91, 1-42.

White, H., 1989. An additional hidden unit test for neglected nonlinearity in multilayer feedforward networks. in Proceedings of the International Joint Conference on Neural Networks. IEEE Press, New York, pp. 451-455. 
Table 1. Simulation Results: Lyapunov Exponent Estimates of Observed Variables

\begin{tabular}{|c|c|c|c|c|c|}
\hline \multicolumn{6}{|c|}{ (A) Logistic Map } \\
\hline$\sigma$ & 0.00 & 0.05 & 0.10 & 0.20 & 0.50 \\
\hline true $\lambda$ & 0.693 & 0.693 & 0.693 & 0.694 & 0.699 \\
\hline$T=50$ & $\begin{array}{c}0.691 / 0.692 \\
(0.023)\end{array}$ & $\begin{array}{c}0.693 / 0.693 \\
(0.025)\end{array}$ & $\begin{array}{c}0.694 / 0.693 \\
(0.027)\end{array}$ & $\begin{array}{c}0.695 / 0.695 \\
(0.026)\end{array}$ & $\begin{array}{c}0.699 / 0.699 \\
(0.032)\end{array}$ \\
\hline 100 & $\begin{array}{c}0.694 / 0.693 \\
(0.014)\end{array}$ & $\begin{array}{c}0.693 / 0.693 \\
(0.013)\end{array}$ & $\begin{array}{c}0.693 / 0.693 \\
(0.013)\end{array}$ & $\begin{array}{c}0.695 / 0.694 \\
(0.014)\end{array}$ & $\begin{array}{c}0.699 / 0.699 \\
(0.018)\end{array}$ \\
\hline 200 & $\begin{array}{c}0.693 / 0.693 \\
(0.006)\end{array}$ & $\begin{array}{c}0.693 / 0.693 \\
\quad(0.007)\end{array}$ & $\begin{array}{c}0.693 / 0.693 \\
\quad(0.007)\end{array}$ & $\begin{array}{c}0.694 / 0.694 \\
\quad(0.008)\end{array}$ & $\begin{array}{c}0.699 / 0.699 \\
\quad(0.011)\end{array}$ \\
\hline 400 & $\begin{array}{c}0.693 / 0.693 \\
\quad(0.003)\end{array}$ & $\begin{array}{c}0.693 / 0.693 \\
\quad(0.004)\end{array}$ & $\begin{array}{c}0.693 / 0.693 \\
(0.004)\end{array}$ & $\begin{array}{c}0.694 / 0.694 \\
\quad(0.004)\end{array}$ & $\begin{array}{c}0.699 / 0.699 \\
\quad(0.006)\end{array}$ \\
\hline 800 & $\begin{array}{c}0.693 / 0.693 \\
(0.002)\end{array}$ & $\begin{array}{c}0.693 / 0.693 \\
(0.002) \\
\end{array}$ & $\begin{array}{c}0.693 / 0.693 \\
(0.002)\end{array}$ & $\begin{array}{c}0.694 / 0.694 \\
(0.002)\end{array}$ & $\begin{array}{c}0.699 / 0.699 \\
(0.004)\end{array}$ \\
\hline & & (B) $\mathrm{S}$ & TAR Model & & \\
\hline$\sigma$ & 0.05 & 0.10 & 0.20 & 0.50 & 1.00 \\
\hline true $\lambda$ & -0.077 & -0.159 & -0.286 & -0.460 & -0.561 \\
\hline$T=50$ & $\begin{array}{c}-0.289 /-0.251 \\
(0.169)\end{array}$ & $\begin{array}{c}-0.367 /-0.328 \\
(0.187)\end{array}$ & $\begin{array}{c}-0.501 /-0.467 \\
(0.202)\end{array}$ & $\begin{array}{c}-0.686 /-0.659 \\
(0.244)\end{array}$ & $\begin{array}{c}-0.803 /-0.758 \\
(0.295)\end{array}$ \\
\hline 100 & $\begin{array}{c}-0.168 /-0.154 \\
(0.076)\end{array}$ & $\begin{array}{c}-0.249 /-0.232 \\
(0.093)\end{array}$ & $\begin{array}{c}-0.393 /-0.372 \\
(0.124)\end{array}$ & $\begin{array}{c}-0.586 /-0.565 \\
(0.165)\end{array}$ & $\begin{array}{c}-0.694 /-0.671 \\
(0.194)\end{array}$ \\
\hline 200 & $\begin{array}{c}-0.114 /-0.107 \\
(0.036)\end{array}$ & $\begin{array}{c}-0.193 /-0.186 \\
(0.047)\end{array}$ & $\begin{array}{c}-0.329 /-0.315 \\
(0.071)\end{array}$ & $\begin{array}{c}-0.516 /-0.499 \\
(0.106)\end{array}$ & $\begin{array}{c}-0.621 /-0.603 \\
(0.129)\end{array}$ \\
\hline 400 & $\begin{array}{c}-0.092 /-0.090 \\
(0.019)\end{array}$ & $\begin{array}{c}-0.169 /-0.167 \\
(0.026)\end{array}$ & $\begin{array}{c}-0.297 /-0.295 \\
(0.042)\end{array}$ & $\begin{array}{c}-0.479 /-0.474 \\
(0.066)\end{array}$ & $\begin{array}{c}-0.583 /-0.577 \\
(0.080)\end{array}$ \\
\hline 800 & $\begin{array}{c}-0.081 /-0.080 \\
(0.011)\end{array}$ & $\begin{array}{c}-0.157 /-0.155 \\
(0.016)\end{array}$ & $\begin{array}{c}-0.281 /-0.279 \\
(0.026)\end{array}$ & $\begin{array}{c}-0.459 /-0.455 \\
(0.042) \\
\end{array}$ & $\begin{array}{c}-0.562 /-0.559 \\
(0.052)\end{array}$ \\
\hline
\end{tabular}

Notes: The results are based on 1,000 Monte Carlo draws using the design described in the main text. Both sample mean and median of the kernel estimates of the largest Lyapunov exponents are reported (mean/median). Numbers in parentheses are sample standard deviations. 
Table 2. Simulation Results: Lyapunov Exponent Estimates of Unobserved Common Factors $(T=100)$

\begin{tabular}{|c|c|c|c|c|c|}
\hline \multicolumn{6}{|c|}{ (A) Logistic Map } \\
\hline$\sigma$ & 0.00 & 0.05 & 0.10 & 0.20 & 0.50 \\
\hline true $\lambda$ & 0.693 & 0.693 & 0.693 & 0.694 & 0.699 \\
\hline$N=25$ & $\begin{array}{c}0.355 / 0.364 \\
(0.113)\end{array}$ & $\begin{array}{c}0.350 / 0.361 \\
(0.112)\end{array}$ & $\begin{array}{c}0.355 / 0.364 \\
(0.116)\end{array}$ & $\begin{array}{c}0.353 / 0.366 \\
(0.115)\end{array}$ & $\begin{array}{c}0.354 / 0.358 \\
(0.115)\end{array}$ \\
\hline 50 & $\begin{array}{c}0.514 / 0.517 \\
(0.076)\end{array}$ & $\begin{array}{c}0.517 / 0.520 \\
(0.075)\end{array}$ & $\begin{array}{c}0.516 / 0.516 \\
(0.074)\end{array}$ & $\begin{array}{c}0.513 / 0.519 \\
(0.076)\end{array}$ & $\begin{array}{c}0.516 / 0.521 \\
\quad(0.076)\end{array}$ \\
\hline 100 & $\begin{array}{c}0.598 / 0.599 \\
(0.057)\end{array}$ & $\begin{array}{c}0.598 / 0.601 \\
(0.056)\end{array}$ & $\begin{array}{c}0.597 / 0.599 \\
(0.055)\end{array}$ & $\begin{array}{c}0.596 / 0.598 \\
(0.054)\end{array}$ & $\begin{array}{c}0.608 / 0.610 \\
(0.056)\end{array}$ \\
\hline 200 & $\begin{array}{c}0.645 / 0.645 \\
(0.044)\end{array}$ & $\begin{array}{c}0.643 / 0.644 \\
\quad(0.043)\end{array}$ & $\begin{array}{c}0.643 / 0.644 \\
(0.046)\end{array}$ & $\begin{array}{c}0.643 / 0.645 \\
(0.044)\end{array}$ & $\begin{array}{c}0.649 / 0.648 \\
\quad(0.045)\end{array}$ \\
\hline 400 & $\begin{array}{c}0.668 / 0.668 \\
(0.036)\end{array}$ & $\begin{array}{c}0.668 / 0.668 \\
\quad(0.038)\end{array}$ & $\begin{array}{c}0.666 / 0.667 \\
(0.037)\end{array}$ & $\begin{array}{c}0.670 / 0.671 \\
(0.036)\end{array}$ & $\begin{array}{c}0.674 / 0.675 \\
\quad(0.037)\end{array}$ \\
\hline$\infty$ & $\begin{array}{c}0.694 / 0.693 \\
(0.014)\end{array}$ & $\begin{array}{c}0.693 / 0.693 \\
\quad(0.013)\end{array}$ & $\begin{array}{c}0.693 / 0.693 \\
(0.013)\end{array}$ & $\begin{array}{c}0.695 / 0.694 \\
(0.014)\end{array}$ & $\begin{array}{c}0.699 / 0.699 \\
(0.018)\end{array}$ \\
\hline & & (B) $S$ & TAR Model & & \\
\hline$\sigma$ & 0.05 & 0.10 & 0.20 & 0.50 & 1.00 \\
\hline true $\lambda$ & -0.077 & -0.159 & -0.286 & -0.460 & -0.561 \\
\hline$N=25$ & $\begin{array}{c}-0.296 /-0.264 \\
(0.136)\end{array}$ & $\begin{array}{c}-0.339 /-0.313 \\
(0.123)\end{array}$ & $\begin{array}{c}-0.453 /-0.433 \\
(0.133)\end{array}$ & $\begin{array}{c}-0.635 /-0.619 \\
(0.171)\end{array}$ & $\begin{array}{c}-0.746 /-0.719 \\
(0.201)\end{array}$ \\
\hline 50 & $\begin{array}{c}-0.231 /-0.209 \\
(0.102)\end{array}$ & $\begin{array}{c}-0.291 /-0.273 \\
(0.103)\end{array}$ & $\begin{array}{c}-0.422 /-0.406 \\
(0.126)\end{array}$ & $\begin{array}{c}-0.612 /-0.588 \\
(0.167)\end{array}$ & $\begin{array}{c}-0.719 /-0.697 \\
(0.194)\end{array}$ \\
\hline 100 & $\begin{array}{c}-0.196 /-0.177 \\
(0.093)\end{array}$ & $\begin{array}{c}-0.268 /-0.251 \\
(0.101)\end{array}$ & $\begin{array}{c}-0.404 /-0.385 \\
(0.122)\end{array}$ & $\begin{array}{c}-0.593 /-0.568 \\
(0.163)\end{array}$ & $\begin{array}{c}-0.700 /-0.672 \\
(0.196)\end{array}$ \\
\hline 200 & $\begin{array}{c}-0.184 /-0.166 \\
(0.089)\end{array}$ & $\begin{array}{c}-0.259 /-0.237 \\
(0.101)\end{array}$ & $\begin{array}{c}-0.401 /-0.380 \\
(0.127)\end{array}$ & $\begin{array}{c}-0.587 /-0.567 \\
(0.163)\end{array}$ & $\begin{array}{c}-0.694 /-0.677 \\
(0.190)\end{array}$ \\
\hline 400 & $\begin{array}{c}-0.169 /-0.154 \\
(0.077)\end{array}$ & $\begin{array}{c}-0.247 /-0.232 \\
(0.090)\end{array}$ & $\begin{array}{c}-0.386 /-0.370 \\
(0.114)\end{array}$ & $\begin{array}{c}-0.579 /-0.569 \\
(0.157)\end{array}$ & $\begin{array}{c}-0.690 /-0.676 \\
(0.189)\end{array}$ \\
\hline$\infty$ & $\begin{array}{c}-0.168 /-0.154 \\
(0.076)\end{array}$ & $\begin{array}{c}-0.249 /-0.232 \\
(0.093)\end{array}$ & $\begin{array}{c}-0.393 /-0.372 \\
(0.124)\end{array}$ & $\begin{array}{c}-0.586 /-0.565 \\
(0.165)\end{array}$ & $\begin{array}{c}-0.694 /-0.671 \\
(0.194)\end{array}$ \\
\hline
\end{tabular}

Notes: The results are based on 1,000 Monte Carlo draws using the design described in the main text. Infeasible estimates from Table 1 are also shown as $N=\infty$ as a benchmark case. Both sample mean and median of the kernel estimates of the largest Lyapunov exponents are reported (mean/median). Numbers in parentheses are sample standard deviations. 
Table 3. Simulation Results: Lyapunov Exponent Estimates of Unobserved Common Factors $(T=200)$

\begin{tabular}{|c|c|c|c|c|c|}
\hline \multicolumn{6}{|c|}{ (A) Logistic Map } \\
\hline$\sigma$ & 0.00 & 0.05 & 0.10 & 0.20 & 0.50 \\
\hline true $\lambda$ & 0.693 & 0.693 & 0.693 & 0.694 & 0.699 \\
\hline$N=25$ & $\begin{array}{c}0.355 / 0.364 \\
(0.103)\end{array}$ & $\begin{array}{c}0.355 / 0.365 \\
(0.102)\end{array}$ & $\begin{array}{c}0.358 / 0.362 \\
(0.098)\end{array}$ & $\begin{array}{c}0.354 / 0.364 \\
(0.102)\end{array}$ & $\begin{array}{c}0.356 / 0.364 \\
(0.100)\end{array}$ \\
\hline 50 & $\begin{array}{c}0.514 / 0.517 \\
(0.057)\end{array}$ & $\begin{array}{c}0.514 / 0.515 \\
\quad(0.013)\end{array}$ & $\begin{array}{c}0.511 / 0.515 \\
(0.013)\end{array}$ & $\begin{array}{c}0.516 / 0.521 \\
(0.014)\end{array}$ & $\begin{array}{c}0.520 / 0.521 \\
\quad(0.018)\end{array}$ \\
\hline 100 & $\begin{array}{c}0.599 / 0.600 \\
(0.038)\end{array}$ & $\begin{array}{c}0.599 / 0.600 \\
(0.039)\end{array}$ & $\begin{array}{c}0.601 / 0.603 \\
(0.040)\end{array}$ & $\begin{array}{c}0.599 / 0.600 \\
(0.040)\end{array}$ & $\begin{array}{c}0.606 / 0.606 \\
(0.040)\end{array}$ \\
\hline 200 & $\begin{array}{c}0.644 / 0.645 \\
(0.031)\end{array}$ & $\begin{array}{c}0.645 / 0.646 \\
\quad(0.031)\end{array}$ & $\begin{array}{c}0.645 / 0.645 \\
(0.032)\end{array}$ & $\begin{array}{c}0.646 / 0.648 \\
(0.031)\end{array}$ & $\begin{array}{c}0.649 / 0.650 \\
\quad(0.030)\end{array}$ \\
\hline 400 & $\begin{array}{c}0.668 / 0.668 \\
(0.025)\end{array}$ & $\begin{array}{c}0.669 / 0.669 \\
\quad(0.026)\end{array}$ & $\begin{array}{c}0.668 / 0.669 \\
\quad(0.027)\end{array}$ & $\begin{array}{c}0.669 / 0.668 \\
\quad(0.025)\end{array}$ & $\begin{array}{c}0.674 / 0.674 \\
\quad(0.026)\end{array}$ \\
\hline$\infty$ & $\begin{array}{c}0.693 / 0.693 \\
(0.006)\end{array}$ & $\begin{array}{c}0.693 / 0.693 \\
(0.007)\end{array}$ & $\begin{array}{c}0.693 / 0.693 \\
(0.007)\end{array}$ & $\begin{array}{c}0.694 / 0.694 \\
(0.008)\end{array}$ & $\begin{array}{c}0.699 / 0.699 \\
(0.011)\end{array}$ \\
\hline & & (B) $S$ & TAR Model & & \\
\hline$\sigma$ & 0.05 & 0.10 & 0.20 & 0.50 & 1.00 \\
\hline true $\lambda$ & -0.077 & -0.159 & -0.286 & -0.460 & -0.561 \\
\hline$N=25$ & $\begin{array}{c}-0.214 /-0.201 \\
(0.070)\end{array}$ & $\begin{array}{c}-0.271 /-0.262 \\
(0.069)\end{array}$ & $\begin{array}{c}-0.394 /-0.380 \\
(0.088)\end{array}$ & $\begin{array}{c}-0.573 /-0.558 \\
(0.118)\end{array}$ & $\begin{array}{c}-0.679 /-0.661 \\
(0.139)\end{array}$ \\
\hline 50 & $\begin{array}{c}-0.161 /-0.151 \\
(0.052)\end{array}$ & $\begin{array}{c}-0.230 /-0.222 \\
(0.058)\end{array}$ & $\begin{array}{c}-0.359 /-0.348 \\
(0.080)\end{array}$ & $\begin{array}{c}-0.542 /-0.529 \\
(0.113)\end{array}$ & $\begin{array}{c}-0.647 /-0.632 \\
(0.133)\end{array}$ \\
\hline 100 & $\begin{array}{c}-0.141 /-0.135 \\
(0.044)\end{array}$ & $\begin{array}{c}-0.215 /-0.207 \\
(0.049)\end{array}$ & $\begin{array}{c}-0.345 /-0.336 \\
(0.070)\end{array}$ & $\begin{array}{c}-0.529 /-0.515 \\
(0.105)\end{array}$ & $\begin{array}{c}-0.634 /-0.617 \\
(0.125)\end{array}$ \\
\hline 200 & $\begin{array}{c}-0.127 /-0.119 \\
(0.041)\end{array}$ & $\begin{array}{c}-0.203 /-0.197 \\
(0.049)\end{array}$ & $\begin{array}{c}-0.338 /-0.327 \\
(0.073)\end{array}$ & $\begin{array}{c}-0.524 /-0.511 \\
(0.105)\end{array}$ & $\begin{array}{c}-0.628 /-0.615 \\
(0.125)\end{array}$ \\
\hline 400 & $\begin{array}{c}-0.119 /-0.113 \\
(0.037)\end{array}$ & $\begin{array}{c}-0.196 /-0.188 \\
(0.045)\end{array}$ & $\begin{array}{c}-0.330 /-0.322 \\
(0.069)\end{array}$ & $\begin{array}{c}-0.517 /-0.504 \\
(0.103)\end{array}$ & $\begin{array}{c}-0.621 /-0.603 \\
(0.122)\end{array}$ \\
\hline$\infty$ & $\begin{array}{c}-0.114 /-0.107 \\
(0.036)\end{array}$ & $\begin{array}{c}-0.193 /-0.186 \\
(0.047)\end{array}$ & $\begin{array}{c}-0.329 /-0.315 \\
(0.071)\end{array}$ & $\begin{array}{c}-0.516 /-0.499 \\
(0.106)\end{array}$ & $\begin{array}{c}-0.621 /-0.603 \\
(0.129)\end{array}$ \\
\hline
\end{tabular}

Notes: The results are based on 1,000 Monte Carlo draws using the design described in the main text. Infeasible estimates from Table 1 are also shown as $N=\infty$ as a benchmark case. Both sample mean and median of the kernel estimates of the largest Lyapunov exponents are reported (mean/median). Numbers in parentheses are sample standard deviations. 
Table 4. Simulation Results: Lyapunov Exponent Estimates of Unobserved Common Factors $(\mathrm{T}=400)$

\begin{tabular}{|c|c|c|c|c|c|}
\hline \multicolumn{6}{|c|}{ (A) Logistic Map } \\
\hline$\sigma$ & 0.00 & 0.05 & 0.10 & 0.20 & 0.50 \\
\hline true $\lambda$ & 0.693 & 0.693 & 0.693 & 0.694 & 0.699 \\
\hline$N=25$ & $\begin{array}{c}0.358 / 0.370 \\
(0.087)\end{array}$ & $\begin{array}{c}0.357 / 0.372 \\
(0.085)\end{array}$ & $\begin{array}{c}0.358 / 0.369 \\
(0.088)\end{array}$ & $\begin{array}{c}0.357 / 0.369 \\
(0.088)\end{array}$ & $\begin{array}{c}0.359 / 0.369 \\
(0.088)\end{array}$ \\
\hline 50 & $\begin{array}{c}0.516 / 0.519 \\
(0.044)\end{array}$ & $\begin{array}{c}0.515 / 0.518 \\
(0.045)\end{array}$ & $\begin{array}{c}0.513 / 0.516 \\
(0.044)\end{array}$ & $\begin{array}{c}0.514 / 0.515 \\
(0.045)\end{array}$ & $\begin{array}{c}0.519 / 0.523 \\
(0.045)\end{array}$ \\
\hline 100 & $\begin{array}{c}0.602 / 0.602 \\
(0.028)\end{array}$ & $\begin{array}{c}0.601 / 0.602 \\
(0.029)\end{array}$ & $\begin{array}{c}0.603 / 0.604 \\
(0.027)\end{array}$ & $\begin{array}{c}0.600 / 0.602 \\
(0.029)\end{array}$ & $\begin{array}{c}0.605 / 0.605 \\
(0.028)\end{array}$ \\
\hline 200 & $\begin{array}{c}0.646 / 0.646 \\
(0.022)\end{array}$ & $\begin{array}{c}0.646 / 0.647 \\
\quad(0.022)\end{array}$ & $\begin{array}{c}0.646 / 0.646 \\
(0.022)\end{array}$ & $\begin{array}{c}0.646 / 0.646 \\
(0.022)\end{array}$ & $\begin{array}{c}0.651 / 0.651 \\
\quad(0.021)\end{array}$ \\
\hline 400 & $\begin{array}{c}0.668 / 0.669 \\
(0.018)\end{array}$ & $\begin{array}{c}0.670 / 0.670 \\
(0.018)\end{array}$ & $\begin{array}{c}0.668 / 0.668 \\
\quad(0.018)\end{array}$ & $\begin{array}{c}0.671 / 0.671 \\
\quad(0.018)\end{array}$ & $\begin{array}{c}0.675 / 0.674 \\
(0.018)\end{array}$ \\
\hline$\infty$ & $\begin{array}{c}0.693 / 0.693 \\
(0.003)\end{array}$ & $\begin{array}{c}0.693 / 0.693 \\
(0.004)\end{array}$ & $\begin{array}{c}0.693 / 0.693 \\
(0.004)\end{array}$ & $\begin{array}{c}0.694 / 0.694 \\
(0.004)\end{array}$ & $\begin{array}{c}0.699 / 0.699 \\
(0.006)\end{array}$ \\
\hline & & (B) $\mathrm{S}$ & TAR Model & & \\
\hline$\sigma$ & 0.05 & 0.10 & 0.20 & 0.50 & 1.00 \\
\hline true $\lambda$ & -0.077 & -0.159 & -0.286 & -0.460 & -0.561 \\
\hline$N=25$ & $\begin{array}{c}-0.170 /-0.164 \\
(0.039)\end{array}$ & $\begin{array}{c}-0.236 /-0.231 \\
(0.040)\end{array}$ & $\begin{array}{c}-0.356 /-0.350 \\
(0.051)\end{array}$ & $\begin{array}{c}-0.534 /-0.529 \\
(0.073)\end{array}$ & $\begin{array}{c}-0.639 /-0.633 \\
(0.087)\end{array}$ \\
\hline 50 & $\begin{array}{c}-0.134 /-0.130 \\
(0.029)\end{array}$ & $\begin{array}{c}-0.205 /-0.202 \\
(0.032)\end{array}$ & $\begin{array}{c}-0.328 /-0.323 \\
(0.046)\end{array}$ & $\begin{array}{c}-0.508 /-0.501 \\
(0.069)\end{array}$ & $\begin{array}{c}-0.613 /-0.606 \\
(0.082)\end{array}$ \\
\hline 100 & $\begin{array}{c}-0.112 /-0.109 \\
(0.023)\end{array}$ & $\begin{array}{c}-0.187 /-0.185 \\
(0.029)\end{array}$ & $\begin{array}{c}-0.313 /-0.309 \\
(0.045)\end{array}$ & $\begin{array}{c}-0.495 /-0.489 \\
(0.070)\end{array}$ & $\begin{array}{c}-0.600 /-0.591 \\
(0.085)\end{array}$ \\
\hline 200 & $\begin{array}{c}-0.102 /-0.099 \\
(0.022)\end{array}$ & $\begin{array}{c}-0.178 /-0.176 \\
(0.027)\end{array}$ & $\begin{array}{c}-0.305 /-0.302 \\
(0.041)\end{array}$ & $\begin{array}{c}-0.488 /-0.483 \\
(0.064)\end{array}$ & $\begin{array}{c}-0.593 /-0.586 \\
(0.078)\end{array}$ \\
\hline 400 & $\begin{array}{c}-0.098 /-0.094 \\
(0.022)\end{array}$ & $\begin{array}{c}-0.174 /-0.171 \\
(0.028)\end{array}$ & $\begin{array}{c}-0.302 /-0.299 \\
(0.044)\end{array}$ & $\begin{array}{c}-0.482 /-0.476 \\
(0.068)\end{array}$ & $\begin{array}{c}-0.585 /-0.580 \\
(0.082)\end{array}$ \\
\hline$\infty$ & $\begin{array}{c}-0.092 /-0.090 \\
(0.019)\end{array}$ & $\begin{array}{c}-0.169 /-0.167 \\
(0.026)\end{array}$ & $\begin{array}{c}-0.297 /-0.295 \\
(0.042)\end{array}$ & $\begin{array}{c}-0.479 /-0.474 \\
(0.066)\end{array}$ & $\begin{array}{c}-0.583 /-0.577 \\
(0.080) \\
\end{array}$ \\
\hline
\end{tabular}

Notes: The results are based on 1,000 Monte Carlo draws using the design described in the main text. Infeasible estimates from Table 1 are also shown as $N=\infty$ as a benchmark case. Both sample mean and median of the kernel estimates of the largest Lyapunov exponents are reported (mean/median). Numbers in parentheses are sample standard deviations. 
Table 5. Test for Neglected Nonlinearity in Principal Components

\begin{tabular}{|c|c|c|c|c|c|c|c|c|}
\hline & $p=1$ & $\overline{p=2}$ & $p=3$ & $\overline{p=4}$ & $\overline{p=1}$ & $p=2$ & $\overline{p=3}$ & $\overline{p=4}$ \\
\hline & \multicolumn{4}{|c|}{$\begin{array}{c}\text { RESET } \\
\text { (Ramsey, 1969) }\end{array}$} & \multicolumn{4}{|c|}{$\begin{array}{c}\text { NN } \\
\text { (White, 1989) }\end{array}$} \\
\hline$k=1$ & 0.012 & 0.025 & 0.053 & 0.054 & 0.016 & 0.003 & 0.007 & $<0.001$ \\
\hline$k=2$ & 0.191 & 0.101 & 0.361 & 0.350 & 0.210 & $<0.001$ & $<0.001$ & 0.202 \\
\hline$k=3$ & 0.002 & $<0.001$ & $<0.001$ & $<0.001$ & 0.001 & 0.002 & $<0.001$ & $<0.001$ \\
\hline$k=4$ & 0.001 & 0.111 & 0.257 & 0.117 & 0.002 & 0.003 & 0.002 & $<0.001$ \\
\hline$k=5$ & $<0.001$ & $<0.001$ & $<0.001$ & $<0.001$ & $<0.001$ & 0.001 & 0.586 & 0.004 \\
\hline \multirow[t]{2}{*}{$k=6$} & 0.008 & 0.020 & 0.047 & 0.125 & 0.007 & 0.038 & 0.080 & 0.086 \\
\hline & \multicolumn{4}{|c|}{ NN-LM } & \multicolumn{4}{|c|}{$\begin{array}{c}\text { KERNEL } \\
\text { (Fan and Li, 1997) }\end{array}$} \\
\hline$k=1$ & 0.006 & 0.002 & $<0.001$ & $<0.001$ & 0.745 & 0.777 & 0.775 & 0.763 \\
\hline$k=2$ & 0.108 & 0.001 & $<0.001$ & $<0.001$ & 0.804 & 0.814 & 0.816 & 0.832 \\
\hline$k=3$ & 0.001 & $<0.001$ & $<0.001$ & $<0.001$ & 0.775 & 0.762 & 0.776 & 0.797 \\
\hline$k=4$ & 0.001 & 0.007 & 0.002 & $<0.001$ & 0.675 & 0.759 & 0.782 & 0.764 \\
\hline$k=5$ & $<0.001$ & $<0.001$ & $<0.001$ & $<0.001$ & 0.760 & 0.770 & 0.783 & 0.790 \\
\hline$k=6$ & 0.005 & 0.017 & 0.085 & 0.060 & 0.729 & 0.779 & 0.797 & 0.823 \\
\hline
\end{tabular}

Notes: Numbers are $p$-values of the tests for the null hypothesis of linearity applied to $k$-th principal components of 159 macroeconomic variables $(N=159)$. The sample period is 1959:3-1998:12 $(T=478)$. 
Table 6. Lyapunov Exponent Estimates of Principal Components

\begin{tabular}{|c|c|c|c|c|c|c|c|c|}
\hline & \multicolumn{4}{|c|}{ (1) Full Sample } & \multicolumn{4}{|c|}{ (2) Block } \\
\hline & $p=1$ & $p=2$ & $p=3$ & $p=4$ & $p=1$ & $p=2$ & $p=3$ & $p=4$ \\
\hline \multicolumn{9}{|c|}{ (A) Kernel Estimation } \\
\hline \multirow[t]{2}{*}{$k=1$} & -0.527 & -0.321 & -0.223 & -0.160 & -0.540 & -0.311 & -0.235 & -0.139 \\
\hline & $(0.018)$ & $(0.027)$ & $(0.017)$ & $(0.015)$ & $(0.038)$ & $(0.047)$ & $(0.046)$ & $(0.028)$ \\
\hline \multirow[t]{2}{*}{$k=2$} & -0.239 & -0.120 & -0.077 & -0.082 & -0.239 & -0.095 & -0.069 & -0.071 \\
\hline & $(0.010)$ & $(0.015)$ & $(0.008)$ & $(0.007)$ & $(0.017)$ & $(0.008)$ & $(0.009)$ & $(0.013)$ \\
\hline \multirow[t]{2}{*}{$k=3$} & -0.998 & -0.837 & -0.512 & -0.391 & -1.019 & -0.763 & -0.515 & -0.393 \\
\hline & $(0.020)$ & $(0.028)$ & $(0.021)$ & $(0.019)$ & $(0.052)$ & $(0.058)$ & $(0.047)$ & $(0.052)$ \\
\hline \multirow{2}{*}{$k=4$} & -2.283 & -0.906 & -0.315 & -0.184 & -2.252 & -0.868 & -0.327 & -0.182 \\
\hline & $(0.063)$ & $(0.017)$ & $(0.011)$ & $(0.009)$ & $(0.159)$ & $(0.054)$ & $(0.025)$ & $(0.019)$ \\
\hline \multirow{2}{*}{$k=5$} & -1.423 & -1.003 & -0.770 & -0.481 & -1.413 & -0.983 & -0.753 & -0.447 \\
\hline & $(0.011)$ & $(0.021)$ & $(0.020)$ & $(0.017)$ & $(0.024)$ & $(0.058)$ & $(0.047)$ & $(0.052)$ \\
\hline \multirow[t]{2}{*}{$k=6$} & -0.722 & -0.290 & -0.146 & -0.105 & -0.715 & -0.311 & -0.150 & -0.101 \\
\hline & $(0.034)$ & $(0.018)$ & $(0.009)$ & $(0.007)$ & $(0.077)$ & $(0.057)$ & $(0.020)$ & $(0.020)$ \\
\hline \multicolumn{9}{|c|}{ (B) Neural Network Estimation } \\
\hline \multirow[t]{2}{*}{$k=1$} & -0.515 & -0.267 & -0.218 & -0.094 & -0.527 & -0.275 & -0.220 & -0.088 \\
\hline & $(0.020)$ & $(0.024)$ & $(0.026)$ & $(0.020)$ & $(0.055)$ & $(0.069)$ & $(0.053)$ & $(0.042)$ \\
\hline \multirow[t]{2}{*}{$k=2$} & -0.235 & -0.092 & -0.061 & -0.046 & -0.240 & -0.088 & -0.053 & -0.041 \\
\hline & $(0.018)$ & $(0.009)$ & $(0.006)$ & $(0.005)$ & $(0.037)$ & $(0.007)$ & $(0.007)$ & $(0.013)$ \\
\hline \multirow[t]{2}{*}{$k=3$} & -0.888 & -0.504 & -0.415 & -0.312 & -0.953 & -0.552 & -0.415 & -0.275 \\
\hline & $(0.041)$ & $(0.036)$ & $(0.017)$ & $(0.018)$ & $(0.055)$ & $(0.054)$ & $(0.011)$ & $(0.049)$ \\
\hline \multirow{2}{*}{$k=4$} & -2.385 & -0.890 & -0.302 & -0.210 & -2.364 & -0.924 & -0.298 & -0.189 \\
\hline & $(0.079)$ & $(0.026)$ & $(0.019)$ & $(0.013)$ & $(0.246)$ & $(0.032)$ & $(0.040)$ & $(0.025)$ \\
\hline \multirow[t]{2}{*}{$k=5$} & -1.987 & -0.881 & -0.761 & -0.367 & -1.940 & -0.816 & -0.701 & -0.333 \\
\hline & $(0.039)$ & $(0.026)$ & $(0.024)$ & $(0.022)$ & $(0.099)$ & $(0.055)$ & $(0.062)$ & $(0.057)$ \\
\hline \multirow[t]{2}{*}{$k=6$} & -0.628 & -0.275 & -0.111 & -0.081 & -0.593 & -0.274 & -0.116 & -0.084 \\
\hline & $(0.032)$ & $(0.023)$ & $(0.011)$ & $(0.009)$ & $(0.082)$ & $(0.049)$ & $(0.025)$ & $(0.020)$ \\
\hline
\end{tabular}

Notes: The largest Lyapunov exponents of $k$-th principal components of 159 macroeconomic variables $(N=159)$. The sample period is 1959:3-1998:12 $(T=478)$. Numbers in parentheses are HAC standard errors based on QS kernel with optimal bandwidth (see Andrews, 1991). Median values are reported for the subsample estimates (Block). 
Figure 1. First Principal Component

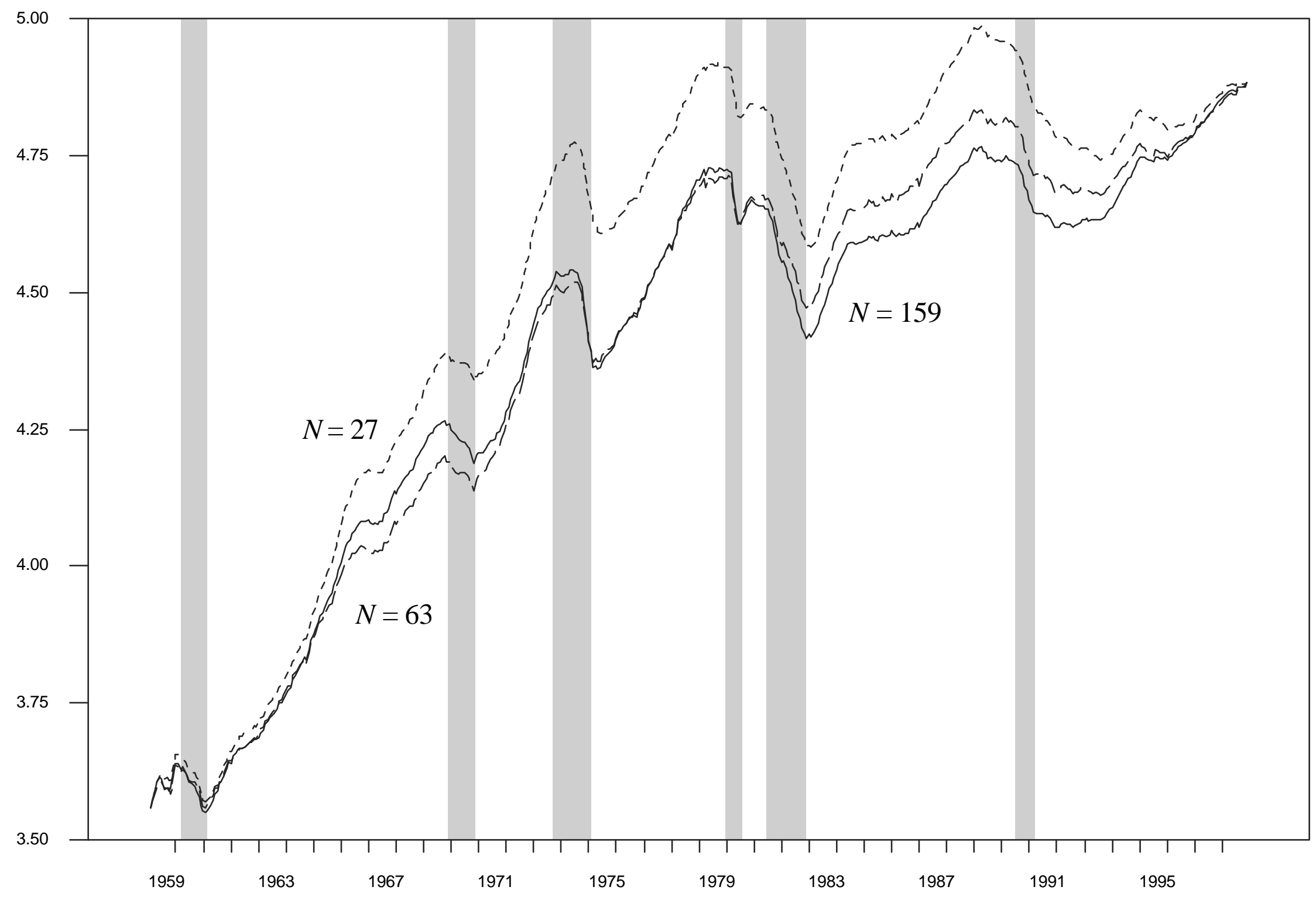

Note: Rescaled using industrial production. 


\section{Figure 2A \\ Nonlinear Impulse Responses \\ [NLAR[1], $k=1]$}

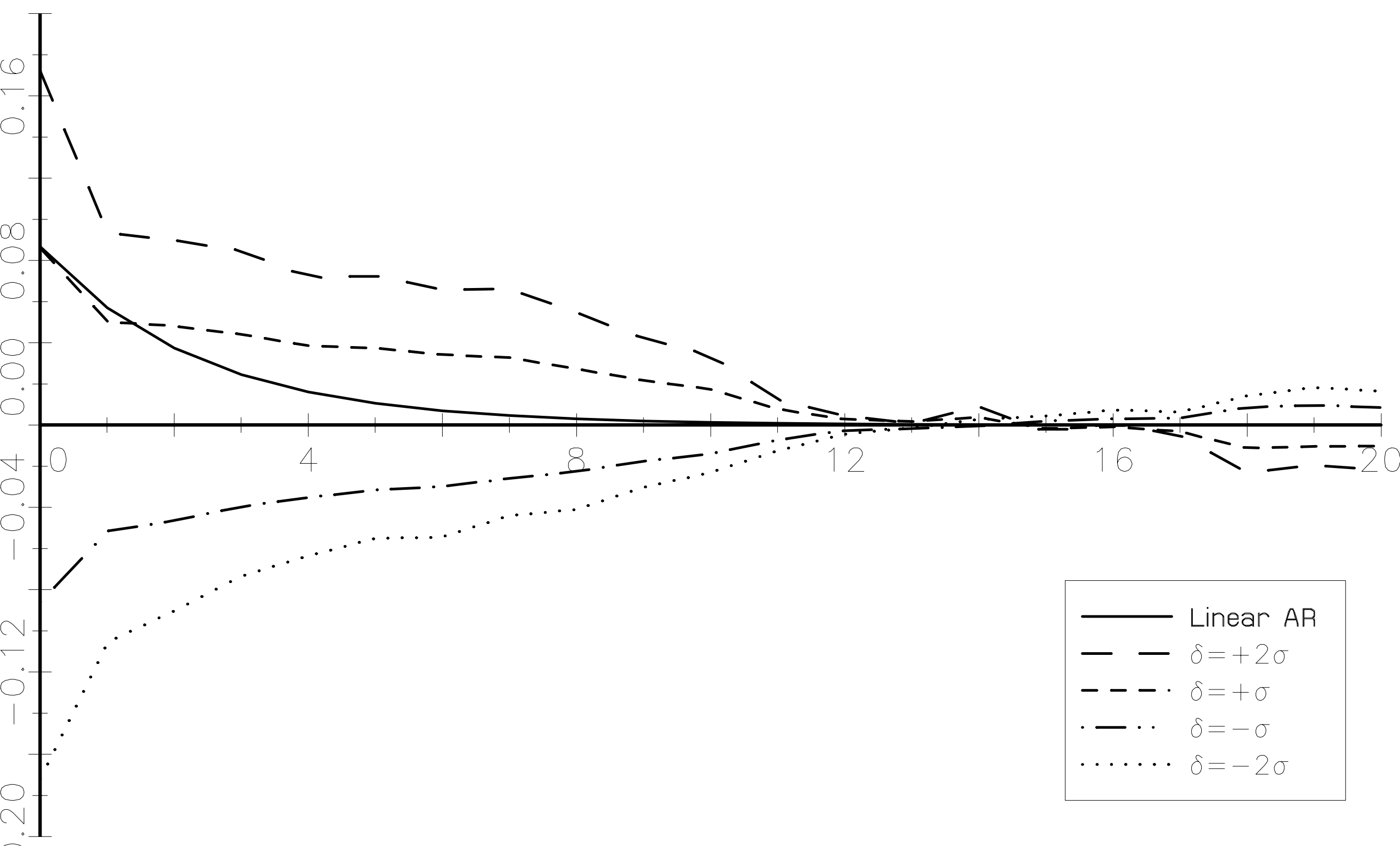




\section{Figure 2B \\ Nonlinear Impulse Responses \\ [NLAR[2], $k=1]$}

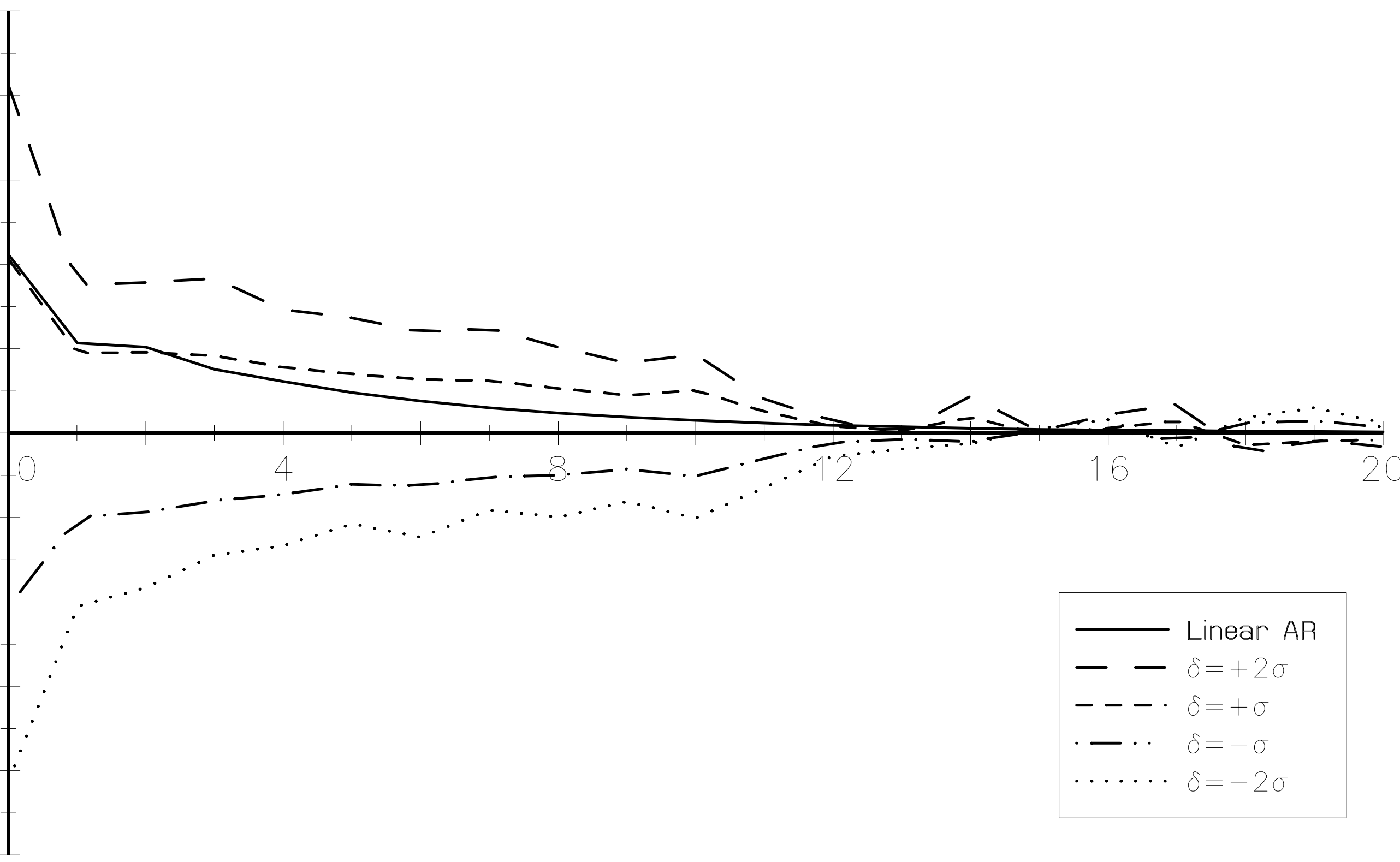


Figure 2C

Nonlinear Impulse Responses

[NLAR[3], $k=1]$






\section{Figure 2D \\ Nonlinear Impulse Responses \\ [NLAR[4], $k=1]$}

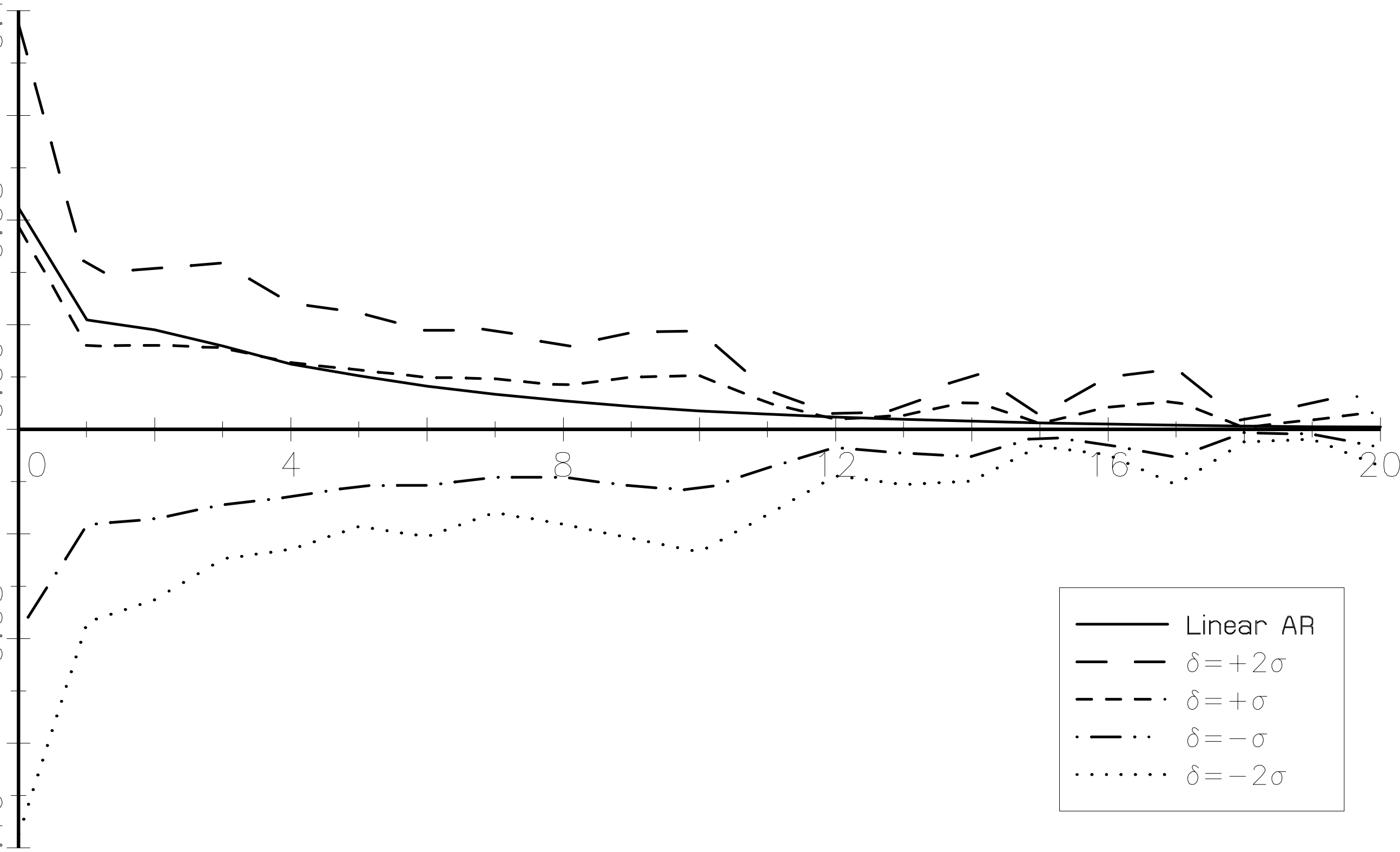




\section{Figure $3 A$ \\ Nonlinear Impulse Responses \\ [NLAR[1], $k=2$ ]}

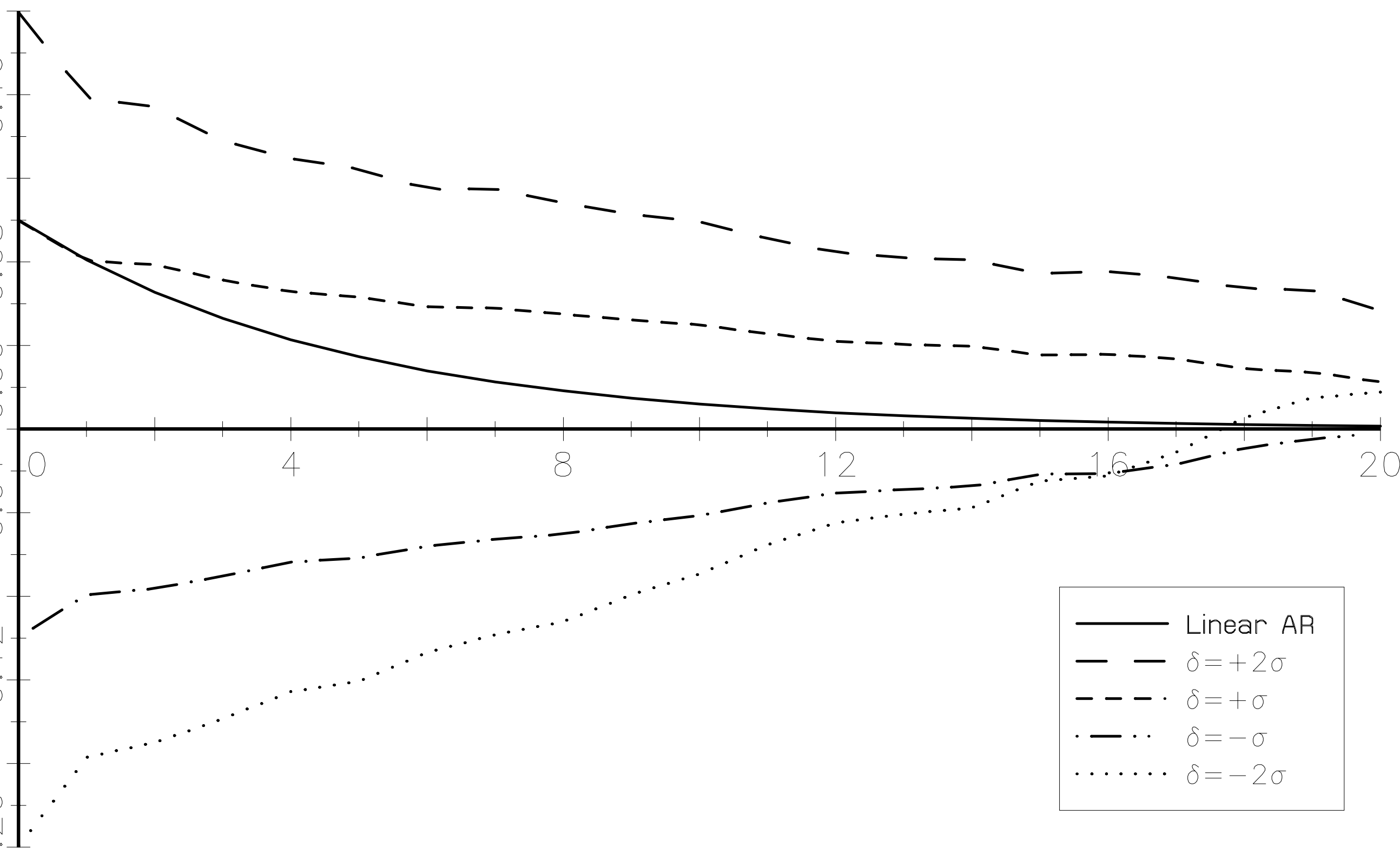


Figure 3B

Nonlinear Impulse Responses

[NLAR[2], $k=2]$

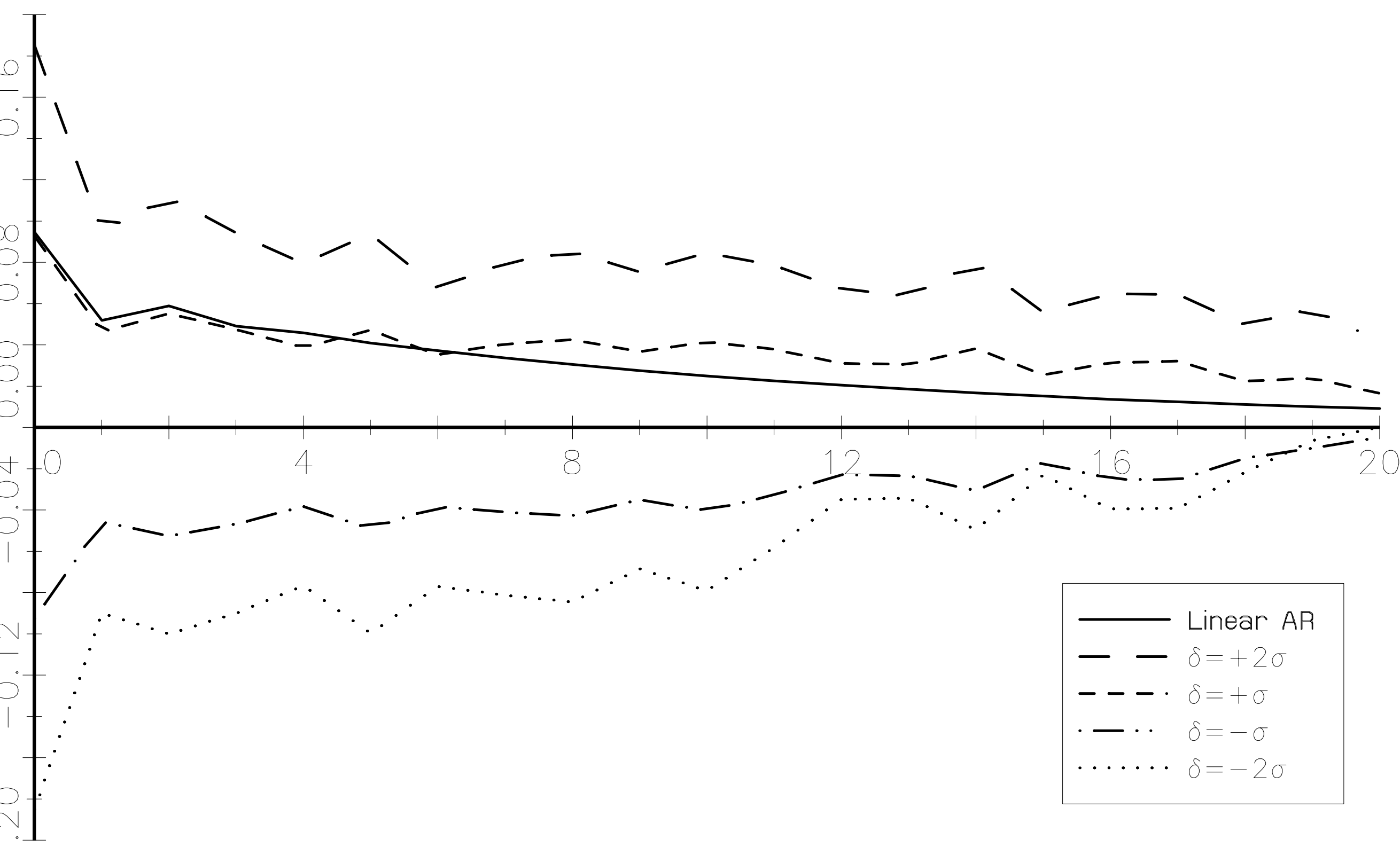




\section{Figure 30 \\ Nonlinear Impulse Responses \\ [NLAR[3], $k=2]$}

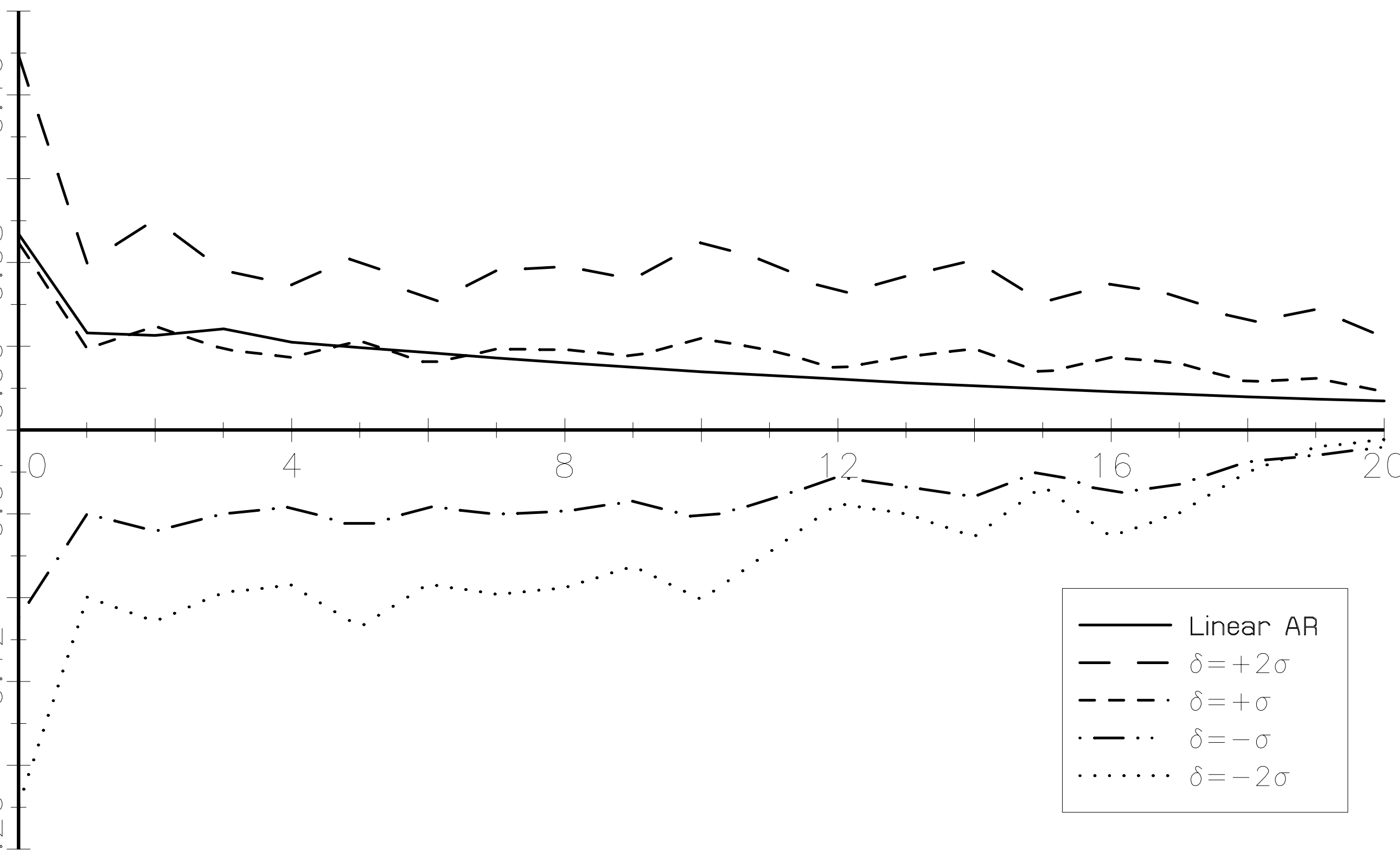




\section{Figure 3D \\ Nonlinear Impulse Responses \\ [NLAR[4], $k=2]$}

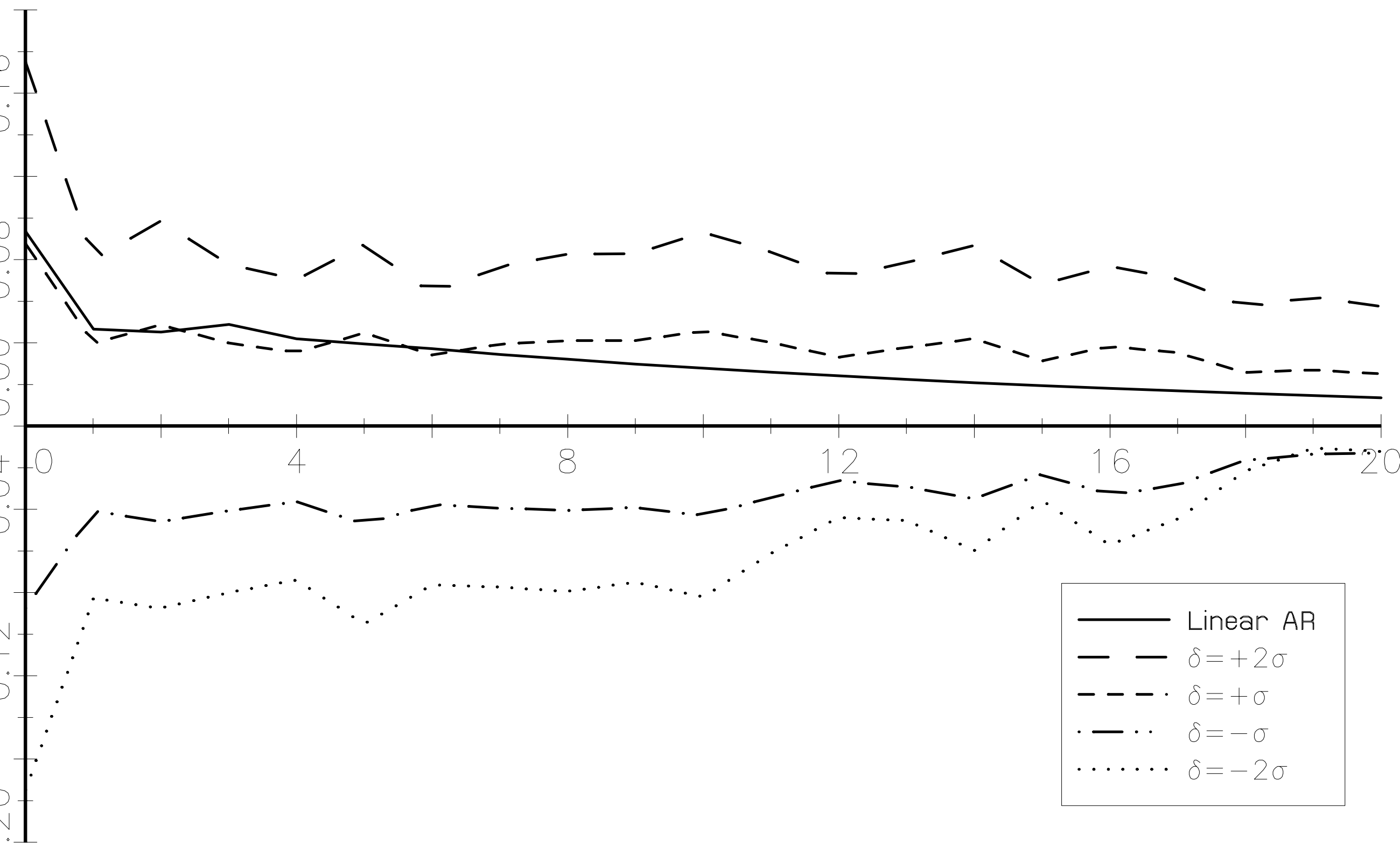

